\title{
Transforming Growth Factor Beta Receptor 3 Haplotypes in Sickle Cell Disease Are Associated with Lipid Profile and Clinical Manifestations
}

\author{
Rayra P. Santiago $\mathbb{D}$, ${ }^{1,2}$ Camylla V. B. Figueiredo $\left(\mathbb{D},{ }^{1,2}\right.$ Luciana M. Fiuza $\mathbb{D},{ }^{1,2}$

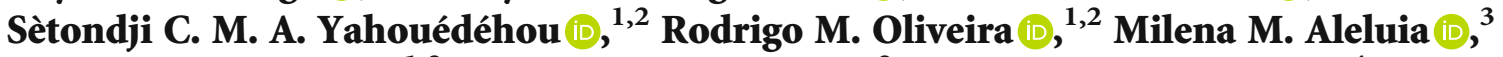 \\ Suellen P. Carvalho $\mathbb{D}^{1,2}$ Cleverson A. Fonseca $\mathbb{D}^{2}{ }^{2}$ Valma M. L. Nascimento, ${ }^{4}$ \\ Larissa C. Rocha, ${ }^{4}$ Caroline C. Guarda $\mathbb{D}^{1,2}$ and Marilda S. Gonçalves $\mathbb{( i D}^{1,2}$ \\ ${ }^{1}$ Laboratório de Investigação em Genética e Hematologia Translacional, Instituto Gonçalo Moniz, FIOCRUZ-BA, \\ Salvador 40296-710, Brazil \\ ${ }^{2}$ Universidade Federal da Bahia, UFBA, Salvador 40170-110, Brazil \\ ${ }^{3}$ Departamento de Ciências Biológicas, Universidade Estadual de Santa Cruz, UESC, Ilhéus 45662-900, Brazil \\ ${ }^{4}$ Fundação de Hematologia e Hemoterapia do Estado da Bahia, HEMOBA, Salvador 40286-240, Brazil \\ Correspondence should be addressed to Marilda S. Gonçalves; mari@bahia.fiocruz.br
}

Received 24 May 2020; Revised 24 August 2020; Accepted 25 September 2020; Published 23 October 2020

Academic Editor: Alex Kleinjan

Copyright (c) 2020 Rayra P. Santiago et al. This is an open access article distributed under the Creative Commons Attribution License, which permits unrestricted use, distribution, and reproduction in any medium, provided the original work is properly cited.

\begin{abstract}
Individuals with sickle cell disease (SCD) present both chronic and acute inflammatory events. The TGF- $\beta$ pathway is known to play a role in immune response, angiogenesis, inflammation, hematopoiesis, vascular inflammation, and cell proliferation. Polymorphisms in the transforming growth factor-beta receptor 3 (TGFBR3) gene have been linked to several inflammatory diseases. This study investigated associations between two TGFBR3 haplotypes and classical laboratory parameters, as well as clinical manifestations, in SCD. We found that individuals with the GG haplotype presented higher levels of total cholesterol (TC), low-density lipoprotein cholesterol (LDL-C), triglycerides, non-HDL cholesterol, total proteins, and globulin than individuals with non-GG haplotypes. In addition, the GG haplotype was associated with a previous history of pneumonia. Individuals with the CGG haplotype presented increased plateletcrit, TC, LDL-C levels, and non-HDL cholesterol. The CCG haplotype was also associated with a previous history of pneumonia. Our findings suggest that individuals with the GG and CGG haplotypes of TGFBR3 present important alterations in lipid profile.
\end{abstract}

\section{Introduction}

Sickle cell disease (SCD) is an autosomal genetic disorder marked by a chronic inflammatory status with acute episodes. The acute condition occurs as a result of vaso-occlusion, persistent cycles of red blood cell sickling and unsickling, and hemolysis, leading to leukocyte and endothelial cell activation that induces the release of cytokines and adhesion molecules. By contrast, the chronic inflammatory status is the result of ischemic and reperfusion processes, which cause endothelial cell and vascular wall damage [1-3].
The clinical complications, which occur frequently in individuals with SCD, are classified as acute or chronic according to the age of affected individuals, yet are not restricted to any specific stage of life $[4,5]$. SCD individuals also present clinical manifestations including vaso-occlusive (VO) and painful crises, pneumonia, cholelithiasis, stroke, priapism, and chronic injury in a variety of organs [6]. One common acute complication in SCD is acute chest syndrome (ACS), characterized by cough, shortness of breath, and signs of hypoxemia that are difficult to distinguish from acute pneumonia [7]. Pulmonary complications in SCA are mostly 
associated with vascular impairment and vasoconstriction, leading to VO [8]. Among the clinical manifestations associated with hemolysis, cholelithiasis, which is related to gallstone formation and gallbladder obstruction, tends to increase in frequency with age [9]. Cholelithiasis occurs due to the accelerated rate of chronic erythrocyte destruction in individuals with SCD. Heme is released by hemolysis and becomes metabolized into bilirubin, which can form insoluble calcium bilirubinate in the bile and precipitate as pigments that form gallstones [10].

The immunological aspects of SCD have been widely studied among individuals with sickle cell anemia (SCA), with high levels of cytokines detected, including interleukin (IL) 4, 6, 8, 10, and tumor necrosis factor-alpha (TNF-a) [11]. In addition, transforming growth factor-beta (TGF- $\beta$ ) and IL-17 were also found to be associated with vascular activation and inflammation based on a direct association with arginase levels [12]. Differences were found in the levels of cytokines (IL1 $\beta$, IL6, TNF-a, and TGF- $\beta$ ), lipid inflammatory mediators (LTB4 and PGE2), and modulators of vascular remodeling (MMP9 and TIMP1) between SCA individuals in steady- and crisis-state, permitting the characterization of these two groups using these parameters [13].

The TGF- $\beta$ pathway is involved in several cellular processes, since signal transduction involves binding with transforming growth factor- (TGF-) beta receptors (TGF $\beta$ RI, TGF $\beta$ RII, or TGF $\beta$ RIII), which activate mothers against decapentaplegic homolog (SMAD) proteins and other mediators. As TGF- $\beta$ is known to activate several mediators, the TGF- $\beta$ pathway is considered to play a role in immune response, angiogenesis, inflammation, hematopoiesis, vascular inflammation, and cell proliferation $[12,14]$.

The transforming growth factor-beta receptor 3 (TGFBR3) gene encodes a receptor of the transforming growth factor-beta (TGF- $\beta$ ) family, the TGF- $\beta$ type III receptor (T $\beta$ RIII), which presents affinity with all three TGF- $\beta$ isoforms [15]. Polymorphisms in gene TGFBR3 have been linked to several diseases, such as Marfan syndrome, bladder cancer, Behçet's disease, and SCD [14, 16-18]. In individuals with SCD, some polymorphisms have been associated with stroke, leg ulcers, priapism, pulmonary hypertension, osteonecrosis, and acute chest syndrome, all severe clinical manifestations [19-25].

Considering the complex mechanisms underlying the pathogenesis of SCD, we sought to investigate associations between TGFBR3 haplotypes and classical laboratory parameters, as well as clinical manifestations.

\section{Materials and Methods}

2.1. Subjects. One hundred seventy-five individuals with SCD (HbSS and HbSC genotypes) were seen at the Bahia Hemotherapy and Hematology Foundation between October 2016 and September 2017. The individuals, 83/175 (47.4\%) of whom were female, had an average age of $14.46 \pm 3.35$ years and a median age of 14 years (interquartile range [IQR]: 1217 years). All individuals with SCD were in steady-state, characterized by the absence of acute crisis in the three months prior to blood collection procedures. None of the patients were undergoing therapy with lipid-lowering agents, such as statins. All individuals or their legal guardians agreed to biological sample collection procedures and signed terms of informed consent. The present research protocol was approved by the Institutional Research Board of the São Rafael Hospital (HSR protocol number: 1400535) and was conducted in compliance with the Declaration of Helsinki (1964) and its subsequent amendments. Biochemical, hematological, genetic, and immunological analyses were performed at the Clinical Analyses Laboratory of the College of Pharmaceutical Sciences, Federal University of Bahia (LACTFAR-UFBA), and at the Laboratory of Genetic Investigation and Translational Hematology at the Gonçalo Moniz Institute-FIOCRUZ (LIGHT-IGM/FIOCRUZ).

2.2. Clinical Manifestations. All legal guardians of the individuals with SCD were asked to complete a questionnaire containing information on clinical data regarding the occurrence of previous clinical manifestations. All information provided was confirmed by individual patient medical records.

2.3. Hematological and Biochemical Parameters. Blood samples were collected by HEMOBA staff following a fasting period of no less than 12 hours.

Hematological parameters were determined using a Beckman Coulter LH 780 Hematology Analyzer (Beckman Coulter, Brea, California, USA), and hemoglobin profile was confirmed by high-performance liquid chromatography using an HPLC/Variant-II hemoglobin testing system (BioRad, Hercules, California, USA).

An automated A25 chemistry analyzer (Biosystems S.A, Barcelona, Catalunya, Spain) was used to determine biochemical parameters, including total bilirubin and fractions, lactate dehydrogenase $(\mathrm{LDH})$, total protein and fractions, iron, hepatic metabolism, and renal profile. Ferritin levels were measured using an Access 2 Immunochemistry System (Beckman Coulter Inc., Pasadena, California, USA). Creactive protein (CRP) and alpha-1 antitrypsin (AAT) levels were measured using an IMMAGE ${ }^{\circledR}$ Immunochemistry System (Beckman Coulter Inc., Pasadena, California, USA).

2.4. Lipid Profile. Total cholesterol (TC), high-density lipoprotein cholesterol (HDL-C), and triglyceride levels were determined using an A25 chemistry analyzer (Biosystems S.A, Barcelona, Catalunya, Spain), while LDL-C and VLDL$\mathrm{C}$ levels were determined by the Friedewald equation [26]. TC/HDL-C, LDL-C/HDL-C, and triglyceride/HDL-C ratios were calculated to evaluate cardiovascular risk [27-31]. In addition, non-HDL-C was calculated by TC-HDL-C [32].

2.5. Genotype Analysis. A QIAamp DNA Blood Mini Kit (QIAGEN, Hilden, Westphalia, Germany) was used to extract genomic DNA from peripheral blood in accordance with the manufacturer's recommendations. Genotyping of TGFBR3 polymorphisms (rs1805110, rs2038931, rs2765888, rs284157, rs284875, and rs7526590) was performed by TaqMan SNP Genotyping Assays (Applied Biosystems, Foster City, CA) on a 7500 Fast Real-Time PCR System (Applied Biosystems, Foster City, CA). 
2.6. Linkage Disequilibrium Analysis. Haploview software (version 4.2) was used to calculate the linkage disequilibrium (LD) between each pairwise combination of SNPs and haplotype frequencies [33].

2.7. Statistical Analysis. The Statistical Package for the Social Sciences (SPSS) v. 20.0 software (IBM, Armonk, New York, USA) and GraphPad Prism version 6.0 (GraphPad Software, San Diego, California, USA) were used to perform all analyses, with $P$ values $<0.05$ considered significant. $\chi^{2}$ test and Fisher's exact test were used to evaluate associations between polymorphisms and clinical data. Hardy-Weinberg equilibrium (HWE) was assessed using the $\chi^{2}$ test. The ShapiroWilk test was used to determine the distribution of quantitative variables, followed by the Mann-Whitney $U$ test or independent $t$-test to compare two numerical variables according to distribution.

\section{Results}

3.1. Baseline Characteristics of Individuals with SCD. The baseline laboratory parameters of 175 individuals with SCD, expressed as means \pm standard deviation, are shown in Supplementary Table 1. Baseline laboratory characteristics showed that SCD individuals presented anemia, hemolysis, and leukocytosis, together with decreased levels of HDL-C.

3.2. Linkage Disequilibrium and Haplotype Analysis. Genotype frequency testing revealed all six SNPs to be in HWE (Table 1).

Haploview software indicated that all six SNPs met the qualifications for LD analysis, which was performed using the four-gamete rule and solid spine of LD methods. The four-gamete testing method created one block with two SNPs (rs284875 and rs2038931) showing complete LD with $D^{\prime}=1$ and the logarithm of odds ratio (LOD) $<2$ (Figure $1(\mathrm{a})$ ). The haplotype evaluation of this block revealed a higher frequency of the GG haplotype $(f=0.731)$ than the others $(\mathrm{GA}=0.160$ and $\mathrm{AG}=0.109)$ (Figure $1(\mathrm{~b})$ ). LD analysis using the solid spine of LD method created one block with three SNPs (rs2765888, rs284875, and rs2038931) (Figure 2(a)). Haplotype analysis of this block showed a higher frequency of the CGG haplotype $(f=0.621)$ than the others identified $(\mathrm{CGA}=0.160, \mathrm{TGG}=0.110, \mathrm{CAG}=$ 0.093 , and $\mathrm{TAG}=0.016$ ) (Figure 2(b)).

3.3. Association of TGFBR3 rs2765888, rs284875, and rs2038931 Polymorphisms with Laboratory Parameters and Clinical Manifestations in SCD. The dominant genetic model was employed in rs2765888 and rs2038931 polymorphisms, while the recessive genetic model was employed in rs284875 polymorphism to evaluate associations between alleles and laboratory biomarkers. With regard to TGFBR3 rs2765888, individuals with CC genotype presented higher mean corpuscular hemoglobin concentration (MCHC) $(P=0.039)$ and plateletcrit $(P=0.027)$ than those with $\mathrm{T}_{-}$ genotypes (Table 2 ).

Regarding the TGFBR3 rs284875 polymorphism, individuals with GG genotype presented decreased triglyceri-
TABLE 1: Hardy-Weinberg equilibrium values for each TGFBR3 polymorphism.

\begin{tabular}{|c|c|c|c|}
\hline Polymorphism & Frequencies & $\chi^{2}$ & $P$ value \\
\hline \multicolumn{4}{|c|}{ rs1805110 (G > A) } \\
\hline GG & $129 / 175$ & & \\
\hline AG & $44 / 175$ & 0.680 & 0.409 \\
\hline AA & $2 / 175$ & & \\
\hline \multicolumn{4}{|c|}{ rs2038931 (G > A) } \\
\hline GG & $120 / 175$ & & \\
\hline AG & $54 / 175$ & 3.831 & 0.050 \\
\hline AA & $1 / 175$ & & \\
\hline \multicolumn{4}{|c|}{ rs2765888 (C > T) } \\
\hline CC & $133 / 175$ & & \\
\hline $\mathrm{CT}$ & $40 / 175$ & 0.277 & 0.598 \\
\hline TT & $2 / 175$ & & \\
\hline \multicolumn{4}{|l|}{ rs284157 $(\mathrm{C}>\mathrm{T})$} \\
\hline $\mathrm{CC}$ & $73 / 175$ & & \\
\hline $\mathrm{CT}$ & $81 / 175$ & 0.041 & 0.838 \\
\hline TT & $21 / 175$ & & \\
\hline \multicolumn{4}{|c|}{ rs284875 $(\mathrm{A}>\mathrm{G})$} \\
\hline $\mathrm{AA}$ & $4 / 175$ & & \\
\hline AG & $30 / 175$ & 2.289 & 0.130 \\
\hline GG & $141 / 175$ & & \\
\hline \multicolumn{4}{|c|}{$\operatorname{rs} 7526590(\mathrm{~A}>\mathrm{T})$} \\
\hline AA & $118 / 175$ & & \\
\hline AT & $54 / 175$ & 1.300 & 0.254 \\
\hline $\mathrm{T}$ & $3 / 175$ & & \\
\hline
\end{tabular}

des/HDL-C ratio $(P=0.022)$ than those with $A_{-}$genotypes (Table 3).

When the TGFBR3 rs2038931 polymorphism was evaluated individuals with GG genotype presented increased VLDL-C $(P=0.007)$, triglycerides $(P=0.006)$, TC/HDL-C ratio $(P=0.035)$, triglycerides/HDL-C ratio $(P=0.006)$, LDL-C/HDL-C ratio $(P=0.033)$, and total protein $(P=0.030)$ than those with $A_{-}$genotypes (Table 4$)$.

Regarding clinical manifestations, no significant association was found to rs2765888 and rs284875 polymorphisms, while the A_ genotypes of rs2038931 polymorphism was associated with a previous history of cholelithiasis $(P=0.038)$ (Table 5).

3.4. Association of GG Haplotype with Laboratory Parameters and Clinical Manifestations in SCD. A comparison of the hematological, biochemical, and immunological parameters between the GG and non-GG-haplotypes revealed that individuals with the GG haplotype presented higher total cholesterol (TC) $(P=0.019)$, low-density lipoprotein cholesterol (LDL-C) $(P=0.034)$, triglycerides $(P=0.040)$, non-HDL-C $(P=0.022)$, total proteins $(P=0.022)$, and globulin levels $(P=0.046)$ than those with non-GG haplotypes, although statistically higher, serum lipid levels in the GG haplotype are within the normal clinical range (Figure 3) (Table 6). 


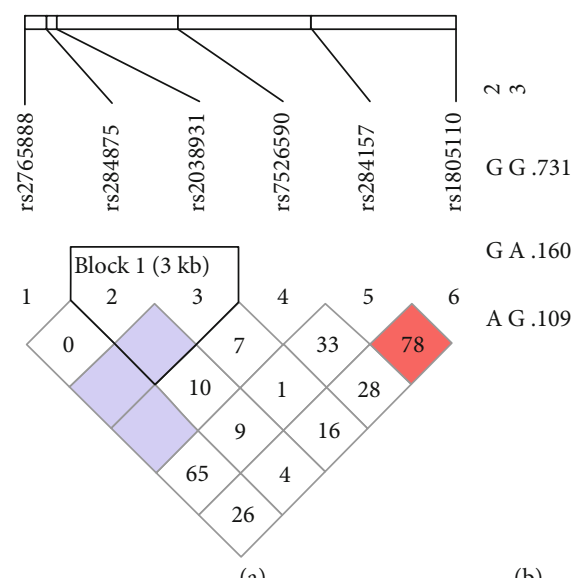

(a)

(b)

FIGURE 1: Linkage disequilibrium analysis of rs2765888 (C > T), rs284875 (A > G), rs2038931 (G > A), rs7526590 (A > T), rs284157 $(\mathrm{C}>\mathrm{T})$, and rs1805110 (G $>\mathrm{A})$ using the four-gamete rule method. (a) According to HaploView 4.2 color schemes, white corresponds to $D^{\prime}<1$ and LOD $<2$, shades of pink/red indicate $D^{\prime}$ $<1$ and $\mathrm{LOD} \geq 2$, and light blue indicates complete $\mathrm{LD}$ with $D^{\prime}=$ 1 and $\mathrm{LOD}<2$. (b) Identified haplotypes and associated frequencies. LOD: Log of odds ratio, a measure of confidence of the value of $D^{\prime}$.

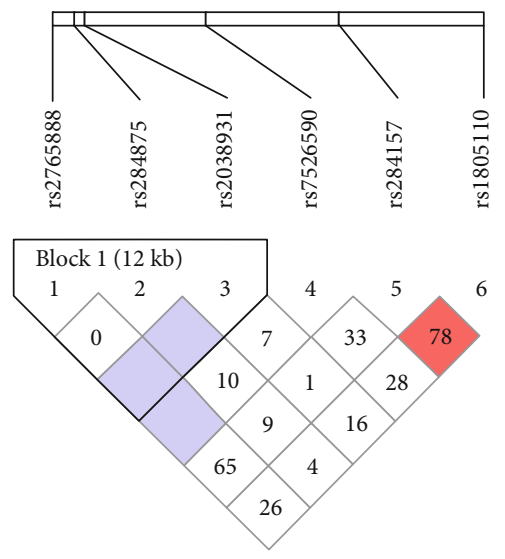

(a) $\rightarrow$ n

C G G .621

C G A .160

T G G .110

C A G .093

T A G .016

(b)
FIGURE 2: Linkage disequilibrium analysis of $\mathrm{rs} 2765888(\mathrm{C}>\mathrm{T})$, rs284875 (A > G), rs2038931 (G > A), rs7526590 (A > T), rs284157 $(C>T)$, and $\operatorname{rs} 1805110(\mathrm{G}>\mathrm{A})$ using the solid spine of $\mathrm{LD}$ method. (a) According to HaploView 4.2 color schemes, white corresponds to $D^{\prime}<1$ and LOD $<2$, shades of pink/red indicate $D^{\prime}$ $<1$ and $L O D \geq 2$, and light blue indicates complete LD with $D^{\prime}=$ 1 and $\mathrm{LOD}<2$. (b) Identified haplotypes and associated frequencies. LOD: Log of odds ratio, a measure of confidence in the value of $D^{\prime}$.

Regarding clinical manifestations, the GG haplotype was associated with a previous history of pneumonia $(P=0.015$ ) (Figure 4(a)), while non-GG haplotypes were associated with a previous history of cholelithiasis $(P=0.021)$ (Figure 4(b)) (Table 5).

3.5. Association of CGG Haplotype with Laboratory Parameters and Clinical Manifestations in SCD. A compari- son of hematological, biochemical, and immunological laboratory parameters between CGG and non-CGG-haplotypes showed that individuals with CGG haplotype presented increased plateletcrit (PCT) $(P=0.046)$, TC $(P=0.029)$, LDL-C $(P=0.035)$, and non-HDL-C $(P=0.030)$ levels, although statistically higher, serum lipid levels in the CGG haplotype are within the normal clinical range (Figure 5) (Table 7). In addition, the CCG haplotype was associated with a previous history of pneumonia $(P=0.018)$ (Figure 4(c)) (Table 5).

3.6. Association of Clinical Manifestations with Laboratory Parameters in SCD. Once the GG and CCG haplotypes were associated with a previous history of pneumonia and the non-GG haplotype was associated with a previous history of cholelithiasis, the association between these clinical manifestations and laboratory parameters was investigated. A comparison of the hematological, biochemical, and immunological parameters between the individuals with and without previous history of pneumonia revealed that individuals with previous history of pneumonia presented higher TC $(P=0.004)$, LDL-C $(P=0.025)$, non-HDL-C $(P=0.012)$, and CRP $(P<0.001)$, as well as decreased creatinine levels $(P=0.014)$ than those without previous history of pneumonia (Table 8).

With regard to cholelithiasis, a comparison of the hematological, biochemical, and immunological parameters between the individuals with and without previous history of this clinical manifestation revealed that individuals with previous history of cholelithiasis presented increased fetal hemoglobin $(P<0.001), S$ hemoglobin $(P=0.002)$, MCV $(P<0.001)$, MCHC $(P<0.001)$, total bilirubin $(P=0.016)$, and indirect bilirubin $(P=0.010)$, as well as decreased red blood cell counts $(P=0.003)$, TC $(P=0.012)$, LDL-C $(P=0.003)$, non-HDL-C $(P=0.007)$, TC/HDL-C ratio $(P<0.001)$, LDL-C/HDL-C ratio $(P<0.001)$, and urea levels $(P=0.002)$ than those without previous history of cholelithiasis (Table 9).

\section{Discussion}

Although polymorphisms in the TGFBR3 gene, when evaluated individually, have been associated with several clinical manifestations in SCD [14, 20-24, 34, 35], Kim et al. (2010) found that, in asthmatic individuals, these polymorphisms are more informative when evaluated in the context of haplotype, versus on an individual basis [36]. Accordingly, this study evaluated associations between TGFBR3 haplotypes and laboratory markers, as well as clinical manifestations, in individuals with SCD.

Laboratory parameters of SCD individuals presented classical markers of hemolysis, anemia, increased leukocyte counts, as well as decreased HDL-C levels. These findings are consistent with previous reports describing SCD individuals [37-40].

In our study, six polymorphisms were evaluated; the polymorphisms rs7526590, rs284875, rs2038931, rs7526590, and rs284157 are intronic variants. Intronic variants might affect alternative splicing of the mRNA; however, until this 
TABLE 2: Association of TGFBR3 rs2765888 polymorphism with laboratory biomarkers using the dominant genetic model.

\begin{tabular}{|c|c|c|c|}
\hline \multirow[b]{2}{*}{ Parameter } & \multicolumn{2}{|c|}{ TGFBR3 rs2765888 polymorphism } & \multirow[b]{2}{*}{$P$ value } \\
\hline & $\begin{array}{c}\text { CC } \\
N=133 \\
\text { Median (IQR) }\end{array}$ & $\begin{array}{c}\mathrm{T}_{-} \\
N=42 \\
\text { Median (IQR) }\end{array}$ & \\
\hline \multicolumn{4}{|l|}{ Hemoglobin pattern } \\
\hline Fetal hemoglobin, \% & $5.70(1.70-10.78)$ & $4.00(1.27-8.60)$ & 0.217 \\
\hline S hemoglobin, \% & $80.90(54.70-90.25)$ & $76.20(52.80-85.58)$ & 0.098 \\
\hline \multicolumn{4}{|l|}{ Hematological markers } \\
\hline RBC, $10^{6} / \mathrm{mL}$ & $2.84(2.49-3.96)$ & $3.29(2.72-3.95)$ & 0.114 \\
\hline Hemoglobin, g/dL & $9.00(7.90-10.80)$ & $9.60(8.20-11.10)$ & 0.219 \\
\hline Hematocrit, \% & $27.00(23.40-32.20)$ & $28.50(25.33-33.00)$ & 0.215 \\
\hline $\mathrm{MCV}, \mathrm{fL}$ & $88.85(80.65-96.33)$ & $85.50(79.80-94.35)$ & 0.361 \\
\hline $\mathrm{MCH}, \rho \mathrm{g}$ & $30.35(27.35-32.80)$ & $28.65(26.60-32.05)$ & 0.146 \\
\hline $\mathrm{MCHC}, \mathrm{g} / \mathrm{dL}$ & $33.90(33.20-34.40)$ & $33.70(33.18-33.90)$ & $0.039^{*}$ \\
\hline RDW, \% & $20.80(18.00-24.20)$ & $20.60(17.43-23.10)$ & 0.515 \\
\hline Reticulocyte count, /mL & $138650(94770-171380)$ & $140025(88680-180015)$ & 0.793 \\
\hline $\mathrm{WBC}, / \mathrm{mL}$ & $10600(8075-13300)$ & $10250(7800-13150)$ & $0.520^{*}$ \\
\hline Neutrophils, /mL & 4687 (3384-6650) & $5550(3550-6518)$ & 0.403 \\
\hline Eosinophils, /mL & $315.00(153.30-578.80)$ & $351.00(155.00-548.00)$ & 0.984 \\
\hline Lymphocytes, /mL & $3834(2833-4601)$ & $3164(2348-4264)$ & 0.050 \\
\hline Monocytes, /mL & $900(600-1313)$ & $818(500-1158)$ & 0.122 \\
\hline Platelet count, $\mathrm{x} 10^{3} / \mathrm{mL}$ & $389.00(289.00-474.00)$ & $332.50(257.00-422.50)$ & 0.071 \\
\hline Platelet volume average, $\mathrm{fL}$ & $7.90(7.40-8.60)$ & $8.10(7.45-8.60)$ & $0.694^{*}$ \\
\hline Plateletcrit, \% & $0.31(0.23-0.37)$ & $0.27(0.20-0.31)$ & 0.027 \\
\hline \multicolumn{4}{|l|}{ Biochemical markers } \\
\hline $\mathrm{TC}, \mathrm{mg} / \mathrm{dL}$ & $124.00(105.00-145.00)$ & $121.00(108.80-134.50)$ & $0.633^{*}$ \\
\hline HDL-C, mg/dL & $36.00(31.00-42.00)$ & $36.50(32.75-43.00)$ & $0.789^{*}$ \\
\hline LDL-C, mg/dL & $64.00(50.60-80.60)$ & $59.00(48.10-77.85)$ & 0.638 \\
\hline VLDL-C, mg/dL & $20.20(14.80-25.40)$ & $19.40(16.50-23.20)$ & 0.964 \\
\hline Triglycerides, $\mathrm{mg} / \mathrm{dL}$ & $101.00(74.00-127.00)$ & $97.00(82.50-116.00)$ & 0.994 \\
\hline Non-HDL-C, mg/dL & $85.50(70.00-104.80)$ & $79.50(69.75-97.75)$ & 0.537 \\
\hline TC/HDL-C ratio & $3.27(2.75-4.14)$ & $3.22(2.66-4.16)$ & 0.739 \\
\hline Triglycerides/HDL-C ratio & $2.57(1.84-3.76)$ & $2.61(1.88-3.46)$ & 0.661 \\
\hline LDL-C/HDL-C ratio & $1.76(1.29-2.32)$ & $1.72(1.18-2.29)$ & 0.828 \\
\hline Total bilirubin, mg/dL & $2.05(1.26-3.13)$ & $1.82(1.21-3.42)$ & 0.704 \\
\hline Direct bilirubin, mg/dL & $0.35(0.26-0.50)$ & $0.35(0.24-0.43)$ & 0.662 \\
\hline Indirect bilirubin, $\mathrm{mg} / \mathrm{dL}$ & $1.63(0.89-2.74)$ & $1.41(0.91-3.12)$ & 0.924 \\
\hline $\mathrm{LDH}, \mathrm{U} / \mathrm{L}$ & $868.00(654.00-1239.00)$ & $847.50(610.80-1265.00)$ & 0.593 \\
\hline ALT, U/L & $15.00(11.00-19.00)$ & $12.00(9.00-20.00)$ & 0.213 \\
\hline AST, U/L & $38.00(26.00-51.25)$ & $34.50(22.75-56.75)$ & 0.418 \\
\hline Total protein, g/dL & $8.19(7.72-8.84)$ & $8.27(7.93-8.90)$ & 0.469 \\
\hline Albumin, g/dL & $4.75(4.57-4.95)$ & $4.86(4.65-5.02)$ & $0.237^{*}$ \\
\hline Globulin, g/dL & $3.48(3.03-4.00)$ & $3.42(3.14-3.94)$ & 0.912 \\
\hline Albumin/globulin ratio & $1.38(1.21-1.58)$ & $1.37(1.25-1.61)$ & $0.679^{*}$ \\
\hline Iron, $\mathrm{mcg} / \mathrm{dL}$ & $92.00(73.75-117.00)$ & $93.00(62.00-134.50)$ & 0.762 \\
\hline Ferritin, $\eta \mathrm{g} / \mathrm{mL}$ & $147.50(90.68-243.80)$ & $121.20(45.10-169.80)$ & 0.221 \\
\hline Urea nitrogen, $\mathrm{mg} / \mathrm{dL}$ & $17.00(13.96-21.00)$ & $18.02(14.31-21.82)$ & 0.201 \\
\hline Creatinine, $\mathrm{mg} / \mathrm{dL}$ & $0.48(0.38-0.58)$ & $0.51(0.39-0.68)$ & 0.147 \\
\hline $\mathrm{CRP}, \mathrm{mg} / \mathrm{L}$ & $2.61(1.73-3.78)$ & $2.39(1.81-5.89)$ & 0.255 \\
\hline $\mathrm{AAT}, \mathrm{mg} / \mathrm{dL}$ & $69.30(37.60-121.30)$ & $72.00(38.00-91.30)$ & 0.886 \\
\hline
\end{tabular}

RBC: red blood cells; MCV: mean cell volume; MCH: mean cell hemoglobin; MCHC: mean corpuscular hemoglobin concentration; RDW: red cell distribution width; LDH: lactate dehydrogenase; WBC: white blood cells; TC: total cholesterol; HDL-C: high-density lipoprotein cholesterol; LDL-C: low-density lipoprotein cholesterol; VLDL-C: very low-density lipoprotein cholesterol; AST: aspartate aminotransferase; ALT: alanine aminotransferase; CRP: C reactive protein; AAT: alpha 1-antitrypsin; IQR: interquartile range. Bold values indicate significance at $P<0.05$. All $P$ values obtained by the Mann-Whitney $U$ test, except for those with asterisk $\left({ }^{*}\right)$, for which the independent $t$-test was used. 
TABLE 3: Association of TGFBR3 rs284875 polymorphism with laboratory biomarkers using the recessive genetic model.

\begin{tabular}{|c|c|c|c|}
\hline \multirow[b]{2}{*}{ Parameter } & \multicolumn{2}{|c|}{ TGFBR3 rs284875 polymorphism } & \multirow[b]{2}{*}{$P$ value } \\
\hline & $\begin{array}{c}\text { GG } \\
N=141 \\
\text { Median (IQR) }\end{array}$ & $\begin{array}{c}\text { A } \\
N=34 \\
\text { Median (IQR) }\end{array}$ & \\
\hline \multicolumn{4}{|l|}{ Hemoglobin pattern } \\
\hline Fetal hemoglobin, \% & $4.70(1.30-10.30)$ & $6.20(2.30-9.80)$ & 0.344 \\
\hline S hemoglobin, \% & $79.90(52.00-88.70)$ & $83.55(56.35-90.13)$ & 0.239 \\
\hline \multicolumn{4}{|l|}{ Hematological markers } \\
\hline $\mathrm{RBC}, 10^{6} / \mathrm{mL}$ & $2.98(2.59-3.98)$ & $2.63(2.40-3.68)$ & 0.090 \\
\hline Hemoglobin, g/dL & $9.10(8.10-11.10)$ & $8.35(7.77-10.35)$ & 0.183 \\
\hline Hematocrit, \% & $27.50(24.00-32.60)$ & $25.70(22.60-31.05)$ & 0.141 \\
\hline $\mathrm{MCV}, \mathrm{fL}$ & $87.10(80.30-94.80)$ & $90.35(82.03-98.30)$ & $0.151^{*}$ \\
\hline $\mathrm{MCH}, \rho \mathrm{g}$ & $29.55(27.20-32.40)$ & $31.15(27.60-34.10)$ & $0.152^{*}$ \\
\hline $\mathrm{MCHC}, \mathrm{g} / \mathrm{dL}$ & $33.70(33.20-34.20)$ & $34.00(33.28-34.53)$ & 0.193 \\
\hline RDW, \% & $20.70(17.90-23.50)$ & $21.70(17.78-25.45)$ & 0.350 \\
\hline Reticulocyte count, $/ \mathrm{mL}$ & $138650(94770-179340)$ & $136010(87075-184758)$ & 0.605 \\
\hline $\mathrm{WBC}, / \mathrm{mL}$ & $10750(8000-13225)$ & $10100(7875-13525)$ & 0.489 \\
\hline Neutrophils, /mL & $5060(3461-6600)$ & 4348 (3386-6677) & 0.413 \\
\hline Eosinophils, /mL & $309.50(153.80-546.00)$ & $396.50(162.50-613.50)$ & 0.671 \\
\hline Lymphocytes, /mL & $3629(2723-4508)$ & 3795 (2375-4656) & 0.825 \\
\hline Monocytes, /mL & $900(600-1296)$ & $810(560-1140)$ & 0.686 \\
\hline Platelet count, $\mathrm{x} 10^{3} / \mathrm{mL}$ & $376.00(278.00-467.00)$ & $388.50(273.30-457.00)$ & $0.909^{*}$ \\
\hline Platelet volume average, $\mathrm{fL}$ & $8.10(7.40-8.60)$ & $7.80(7.37-8.42)$ & $0.256^{*}$ \\
\hline Plateletcrit, \% & $0.29(0.23-0.36)$ & $0.29(0.19-0.36)$ & 0.704 \\
\hline \multicolumn{4}{|l|}{ Biochemical markers } \\
\hline $\mathrm{TC}, \mathrm{mg} / \mathrm{dL}$ & $123.00(108.00-140.00)$ & $118.00(97.00-140.50)$ & 0.296 \\
\hline HDL-C, mg/dL & $37.00(32.00-43.00)$ & $33.00(29.00-41.00)$ & $0.180^{*}$ \\
\hline LDL-C, mg/dL & $64.60(51.55-80.35)$ & $55.20(44.30-75.30)$ & 0.120 \\
\hline VLDL-C, mg/dL & $19.40(14.60-23.60)$ & $21.00(16.00-27.60)$ & 0.091 \\
\hline Triglycerides, mg/dL & $97.00(73.00-118.00)$ & $105.00(80.00-138.00)$ & 0.098 \\
\hline Non-HDL-C, mg/dL & $85.00(72.00-103.00)$ & $81.00(64.50-107.00)$ & 0.489 \\
\hline TC/HDL-C ratio & $3.24(2.76-4.15)$ & $3.29(2.73-4.50)$ & 0.629 \\
\hline Triglycerides/HDL-C ratio & $2.48(1.82-3.50)$ & $2.93(2.31-4.30)$ & 0.022 \\
\hline LDL-C/HDL-C ratio & $1.75(1.33-2.34)$ & $1.78(1.21-2.20)$ & 0.877 \\
\hline Total bilirubin, mg/dL & $2.04(1.24-3.23)$ & $2.00(1.40-3.06)$ & 0.786 \\
\hline Direct bilirubin, mg/dL & $0.35(0.24-0.43)$ & $0.39(0.29-0.54)$ & 0.072 \\
\hline Indirect bilirubin, $\mathrm{mg} / \mathrm{dL}$ & $1.58(0.89-2.90)$ & $1.48(1.01-2.78)$ & 0.886 \\
\hline $\mathrm{LDH}, \mathrm{U} / \mathrm{L}$ & $856.00(630.00-1217.00)$ & $954.00(614.50-1354.00)$ & 0.614 \\
\hline ALT, U/L & $14.00(10.00-19.00)$ & $15.00(9.25-19.50)$ & 0.841 \\
\hline AST, U/L & $37.00(26.00-50.00)$ & $41.00(25.50-57.00)$ & 0.899 \\
\hline Total protein, g/dL & $8.22(7.85-8.85)$ & $8.09(7.51-8.89)$ & 0.427 \\
\hline Albumin, g/dL & $4.76(4.60-4.98)$ & $4.88(4.50-4.99)$ & 0.858 \\
\hline Globulin, g/dL & $3.47(3.11-3.96)$ & $3.25(2.98-4.11)$ & 0.437 \\
\hline Albumin/globulin ratio & $1.36(1.22-1.58)$ & $1.42(1.22-1.61)$ & $0.442^{*}$ \\
\hline Iron, $\mathrm{mcg} / \mathrm{dL}$ & $92.00(72.00-120.00)$ & $92.00(72.50-126.00)$ & 0.921 \\
\hline Ferritin, $\eta \mathrm{g} / \mathrm{mL}$ & $145.70(91.63-214.90)$ & $239.60(50.93-524.70)$ & 0.272 \\
\hline Urea nitrogen, $\mathrm{mg} / \mathrm{dL}$ & $17.00(14.00-21.00)$ & $17.99(14.11-21.07)$ & 0.996 \\
\hline Creatinine, mg/dL & $0.48(0.38-0.60)$ & $0.50(0.34-0.64)$ & 0.962 \\
\hline $\mathrm{CRP}, \mathrm{mg} / \mathrm{L}$ & $2.39(1.71-3.57)$ & $2.66(1.87-4.82)$ & 0.240 \\
\hline $\mathrm{AAT}, \mathrm{mg} / \mathrm{dL}$ & $71.30(37.10-113.00)$ & $69.00(45.20-131.30)$ & 0.444 \\
\hline
\end{tabular}

RBC: red blood cells; MCV: mean cell volume; MCH: mean cell hemoglobin; MCHC: mean corpuscular hemoglobin concentration; RDW: red cell distribution width; LDH: lactate dehydrogenase; WBC: white blood cells; TC: total cholesterol; HDL-C: high-density lipoprotein cholesterol; LDL-C: low-density lipoprotein cholesterol; VLDL-C: very low-density lipoprotein cholesterol; AST: aspartate aminotransferase; ALT: alanine aminotransferase; CRP: C reactive protein; AAT: alpha 1-antitrypsin; IQR: interquartile range. Bold values indicate significance at $P<0.05$. All $P$ values obtained by the Mann-Whitney $U$ test, except for those with asterisk $\left({ }^{*}\right)$, for which the independent $t$-test was used. 
TABLE 4: Association of TGFBR3 rs2038931 polymorphism with laboratory biomarkers using the dominant genetic model.

\begin{tabular}{|c|c|c|c|}
\hline \multirow[b]{2}{*}{ Parameter } & \multicolumn{2}{|c|}{ TGFBR3 rs2038931 polymorphism } & \multirow[b]{2}{*}{$P$ value } \\
\hline & $\begin{array}{c}\text { GG } \\
N=120 \\
\text { Median (IQR) }\end{array}$ & $\begin{array}{c}\text { A } \\
N=55 \\
\text { Median (IQR) }\end{array}$ & \\
\hline \multicolumn{4}{|l|}{ Hemoglobin pattern } \\
\hline Fetal hemoglobin, \% & $4.80(1.70-8.75)$ & $5.50(1.30-12.63)$ & 0.486 \\
\hline S hemoglobin, \% & $80.30(54.50-89.90)$ & $79.95(52.15-87.60)$ & 0.802 \\
\hline \multicolumn{4}{|l|}{ Hematological markers } \\
\hline $\mathrm{RBC}, 10^{6} / \mathrm{mL}$ & $2.96(2.51-3.97)$ & $2.95(2.56-3.79)$ & 0.978 \\
\hline Hemoglobin, g/dL & $9.00(7.90-10.88)$ & $9.20(8.10-10.80)$ & 0.510 \\
\hline Hematocrit, \% & $27.00(23.30-32.65)$ & $27.50(24.30-32.10)$ & 0.524 \\
\hline $\mathrm{MCV}, \mathrm{fL}$ & $87.30(80.10-94.30)$ & $91.20(80.80-97.30)$ & $0.177^{*}$ \\
\hline $\mathrm{MCH}, \rho \mathrm{g}$ & $29.60(27.15-32.65)$ & $30.90(27.60-32.60)$ & $0.270^{*}$ \\
\hline $\mathrm{MCHC}, \mathrm{g} / \mathrm{dL}$ & $33.80(33.20-34.40)$ & $33.70(33.20-34.30)$ & $0.393^{*}$ \\
\hline RDW, \% & $21.30(18.00-24.40)$ & $20.00(17.60-23.00)$ & 0.074 \\
\hline Reticulocyte count, /mL & $131330(88245-174570)$ & $144690(98880-180540)$ & $0.070^{*}$ \\
\hline $\mathrm{WBC}, / \mathrm{mL}$ & $10700(8150-13025)$ & $10450(7550-13625)$ & 0.349 \\
\hline Neutrophils, /mL & $5078(3478-6600)$ & $4402(3356-6675)$ & 0.270 \\
\hline Eosinophils, /mL & $300.00(160.00-544.00)$ & $326.00(142.00-590.00)$ & 0.996 \\
\hline Lymphocytes, /mL & $3702(2783-4601)$ & $3493(2666-4454)$ & 0.339 \\
\hline Monocytes, /mL & $894(600-1297)$ & $800(552-1288)$ & 0.499 \\
\hline Platelet count, $\times 10^{3} / \mathrm{mL}$ & $383.00(278.00-457.00)$ & $372.00(274.00-467.00)$ & 0.951 \\
\hline Platelet volume average, $\mathrm{fL}$ & $7.90(7.50-8.52)$ & $8.10(7.30-8.70)$ & $0.278^{*}$ \\
\hline Plateletcrit, \% & $0.29(0.23-0.36)$ & $0.29(0.22-0.36)$ & 0.877 \\
\hline \multicolumn{4}{|l|}{ Biochemical markers } \\
\hline $\mathrm{TC}, \mathrm{mg} / \mathrm{dL}$ & $126.50(109.30-141.80)$ & $116.00(103.00-137.50)$ & 0.116 \\
\hline HDL-C, mg/dL & $36.00(32.00-42.00)$ & $37.00(32.00-45.00)$ & 0.121 \\
\hline LDL-C, mg/dL & $64.40(50.60-81.40)$ & $58.60(49.90-78.60)$ & 0.236 \\
\hline VLDL-C, mg/dL & $20.50(16.15-25.65)$ & $17.40(12.35-23.20)$ & 0.007 \\
\hline Triglycerides, mg/dL & $103.00(81.00-129.00)$ & $88.00(62.00-116.00)$ & 0.006 \\
\hline Non-HDL-C, mg/dL & $90.00(71.00-106.00)$ & $79.00(67.00-98.50)$ & 0.068 \\
\hline TC/HDL-C ratio & $3.33(2.77-4.35)$ & $3.10(2.67-3.57)$ & 0.035 \\
\hline Triglycerides/HDL-C ratio & $2.69(1.97-3.73)$ & $2.32(1.53-3.10)$ & 0.006 \\
\hline LDL-C/HDL-C ratio & $1.81(1.34-2.43)$ & $1.58(1.15-2.00)$ & 0.033 \\
\hline Total bilirubin, mg/dL & $2.07(1.31-3.06)$ & $1.76(1.15-3.52)$ & 0.654 \\
\hline Direct bilirubin, mg/dL & $0.36(0.24-0.49)$ & $0.35(0.28-0.43)$ & 0.966 \\
\hline Indirect bilirubin, $\mathrm{mg} / \mathrm{dL}$ & $1.60(0.98-2.63)$ & $1.29(0.85-3.12)$ & 0.695 \\
\hline $\mathrm{LDH}, \mathrm{U} / \mathrm{L}$ & $907.50(630.50-1318.00)$ & $841.00(609.00-1085.00)$ & 0.227 \\
\hline ALT, U/L & $14.50(10.75-20.00)$ & $15.00(10.00-19.00)$ & 0.784 \\
\hline AST, U/L & $37.00(26.00-54.00)$ & $38.00(24.00-49.00)$ & 0.627 \\
\hline Total protein, g/dL & $8.27(7.93-8.87)$ & $8.00(7.50-8.66)$ & $0.030^{*}$ \\
\hline Albumin, g/dL & $4.83(4.60-5.00)$ & $4.71(4.53-4.90)$ & $0.066^{*}$ \\
\hline Globulin, g/dL & $3.50(3.11-4.00)$ & $3.30(2.94-3.93)$ & 0.078 \\
\hline Albumin/globulin ratio & $1.37(1.21-1.51)$ & $1.40(1.23-1.64)$ & $0.381^{*}$ \\
\hline Iron, $\mathrm{mcg} / \mathrm{dL}$ & $92.00(72.00-117.00)$ & $92.00(72.50-128.30)$ & 0.483 \\
\hline Ferritin, $\eta \mathrm{g} / \mathrm{mL}$ & $134.00(71.55-189.10)$ & $190.30(89.33-263.00)$ & 0.082 \\
\hline Urea nitrogen, $\mathrm{mg} / \mathrm{dL}$ & $17.22(14.30-20.94)$ & $15.00(13.40-22.00)$ & $0.367^{*}$ \\
\hline Creatinine, $\mathrm{mg} / \mathrm{dL}$ & $0.49(0.36-0.62)$ & $0.48(0.38-0.59)$ & 0.815 \\
\hline $\mathrm{CRP}, \mathrm{mg} / \mathrm{L}$ & $2.40(1.71-3.79)$ & $2.58(1.77-3.51)$ & 0.866 \\
\hline $\mathrm{AAT}, \mathrm{mg} / \mathrm{dL}$ & $68.20(38.08-117.80)$ & $73.80(37.30-120.00)$ & 0.859 \\
\hline
\end{tabular}

RBC: red blood cells; MCV: mean cell volume; MCH: mean cell hemoglobin; MCHC: mean corpuscular hemoglobin concentration; RDW: red cell distribution width; LDH: lactate dehydrogenase; WBC: white blood cells; TC: total cholesterol; HDL-C: high-density lipoprotein cholesterol; LDL-C: low-density lipoprotein cholesterol; VLDL-C: very-low-density lipoprotein cholesterol; AST: aspartate aminotransferase; ALT: alanine aminotransferase; CRP: C reactive protein; AAT: alpha 1-antitrypsin; IQR: interquartile range. Bold values indicate significance at $P<0.05$. All $P$ values obtained by the Mann-Whitney $U$ test, except for those with asterisk $\left({ }^{*}\right)$, for which the independent $t$-test was used. 


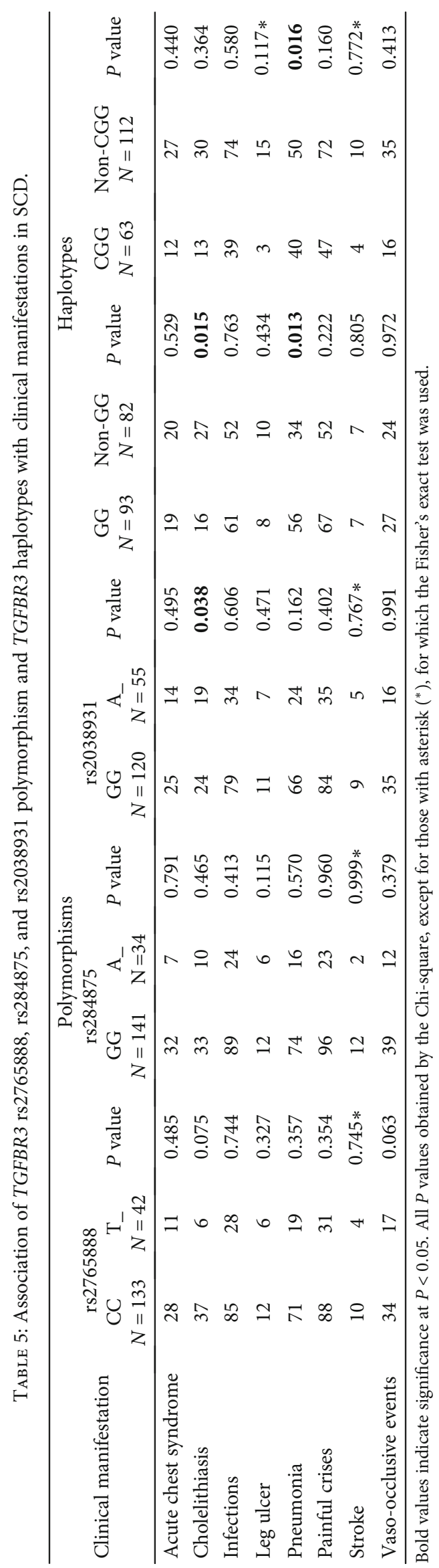




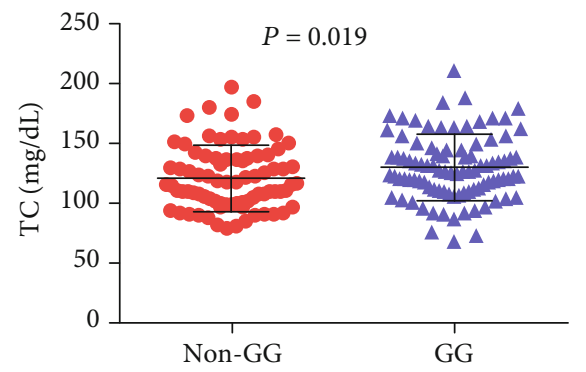

(a)

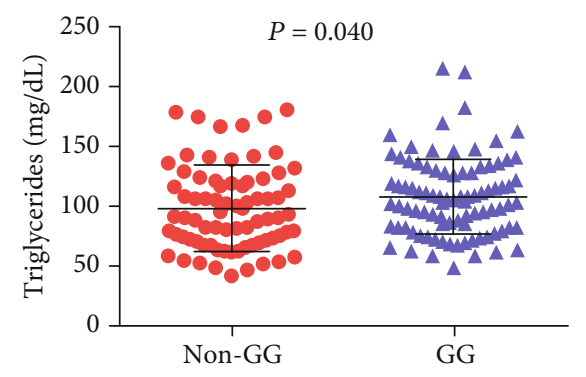

(c)

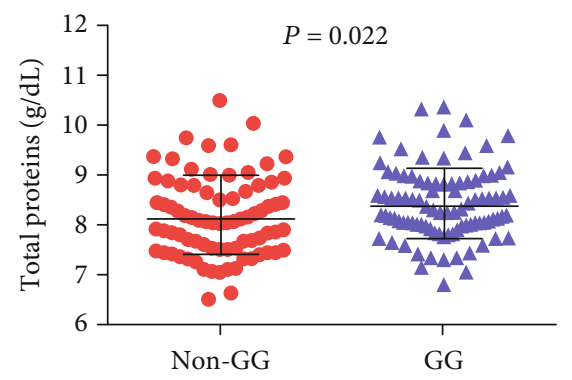

(e)

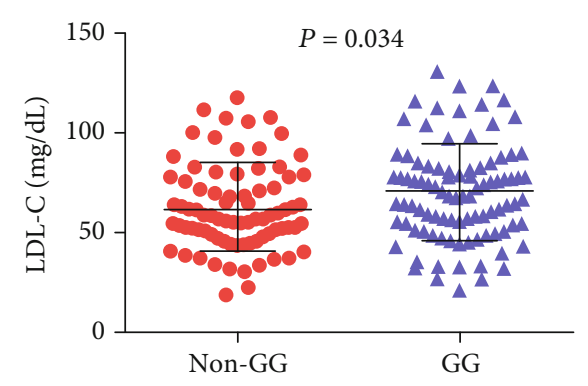

(b)

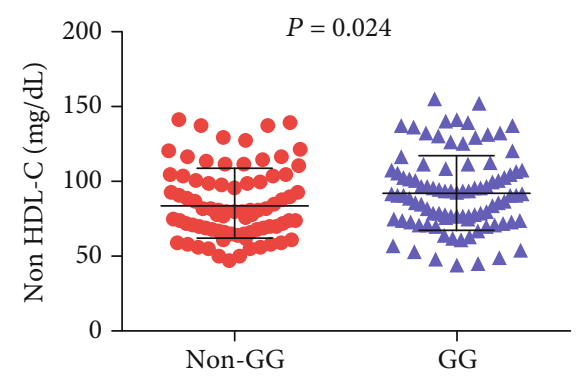

(d)

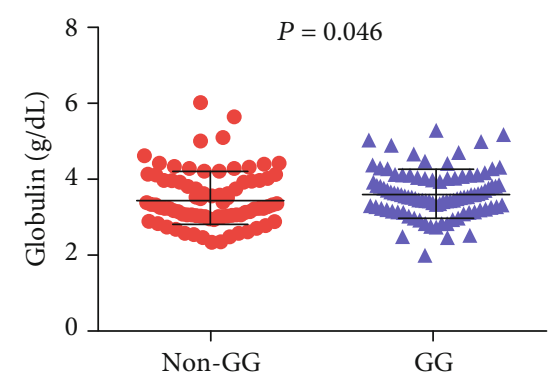

(f)

FIGURE 3: Associations between the GG haplotype and laboratory parameters in individuals with SCD. Carriers of the GG haplotype presented increased (a) total cholesterol (TC) levels, (b) low-density lipoproteins cholesterol (LDL-C) levels, (c) triglycerides, and (d) nonlow-density lipoprotein cholesterol (non-HDL-C) (all $P$ values obtained by the Mann-Whitney $U$ test). Carriers of the GG haplotype also had presented increased (e) total protein levels ( $P$ value obtained with $t$-testing) and (f) increased globulin levels $(P$ value obtained by the Mann-Whitney $U$ test).

moment, there are no evidences of association between these polymorphisms and TGFBR3 function or TGFbeta signaling. We reinforce that all the selected SNPs were previously studied and associated with the occurrence of clinical manifestation in SCD [20-24]. With regard to rs1805110 polymorphism, this is a missense variant that causes a change in the polypeptide chain where a $S(\mathrm{Ser})>F(\mathrm{Phe})$. This polymorphism has been associated with Behçet's disease, susceptibility in HBV-related hepatocellular carcinoma, pulmonary emphysema, and aneurysm; however, it was not previously investigated in SCD [41-44].

Our results showed that individuals with the GG haplotype of TGFBR3 had higher levels of TC, LDL-C, triglycerides, and non-HDL-C than those with non-GG haplotypes. In addition, individuals with the CGG haplotype of TGFBR3 also presented lipid profile alterations, i.e., higher TC, LDL$\mathrm{C}$, and non-HDL-C levels than those with non-CGG haplotypes. Importantly, even statistically higher, serum lipid levels in the GG and CGG haplotypes were within the normal clinical range. The individual evaluation of polymorphisms showed that individuals with GG genotype of rs2038931 polymorphism presented increased VLDL-C, triglycerides, TC/HDL-C ratio, triglycerides/HDL-C ratio, and LDL$\mathrm{C} / \mathrm{HDL}-\mathrm{C}$ ratio than those with $\mathrm{A}_{-}$genotypes. Moreover, the CC genotype of rs2765888 polymorphism was also associated with increased triglycerides/HDL-C ratio. Indeed, when the TGFBR3 rs2765888, rs284875, and rs2038931 polymorphisms, which compose the haplotypes, were individually investigated, none of them seems to contribute more than the other polymorphism to the associations found by the haplotypes. Thus, the associations between the haplotypes and lipid parameters were equally modulated by the polymorphisms. Despite the rs2038931 polymorphism has been associated with lipid markers, they were different from those identified by haplotypes.

High total cholesterol, as well as LDL-C levels, triggers the process of atherosclerosis by stimulating cholesterol accumulation and promotes an inflammatory response in the artery wall [45]. The pathophysiological mechanisms present in atherosclerosis are analogous to those identified 
TABLE 6: Association of TGFBR3 GG haplotype with laboratory biomarkers.

\begin{tabular}{|c|c|c|c|}
\hline \multirow[b]{2}{*}{ Parameter } & \multicolumn{2}{|c|}{ TGFBR3 GG haplotype } & \multirow[b]{2}{*}{$P$ value } \\
\hline & $\begin{array}{c}\text { GG } \\
N=93 \\
\text { Median (IQR) }\end{array}$ & $\begin{array}{c}\text { Non-GG } \\
N=82 \\
\text { Median (IQR) }\end{array}$ & \\
\hline \multicolumn{4}{|l|}{ Hemoglobin pattern } \\
\hline Fetal hemoglobin, \% & $80.10(52.08-89.88)$ & $80.20(54.50-88.60)$ & 0.688 \\
\hline S hemoglobin, \% & $4.50(1.40-8.60)$ & $6.20(1.70-11.600)$ & 0.142 \\
\hline \multicolumn{4}{|l|}{ Hematological markers } \\
\hline $\mathrm{RBC}, 10^{6} / \mathrm{mL}$ & $2.98(2.57-3.98)$ & $2.90(2.49-3.71)$ & 0.345 \\
\hline Hemoglobin, g/dL & $9.10(8.00-11.10)$ & $9.10(8.10-10.73)$ & 0.800 \\
\hline Hematocrit, \% & $27.00(23.90-33.00)$ & $27.35(23.48-32.10)$ & 0.765 \\
\hline $\mathrm{MCV}, \mathrm{fL}$ & $86.20(80.15-93.85)$ & $90.70(80.78-97.50)$ & $0.063^{*}$ \\
\hline $\mathrm{MCH}, \rho \mathrm{g}$ & $29.10(27.03-32.33)$ & $30.95(27.55-32.85)$ & $0.778^{*}$ \\
\hline $\mathrm{MCHC}, \mathrm{g} / \mathrm{dL}$ & $33.70(33.20-34.20)$ & $33.80(33.20-34.40)$ & 0.819 \\
\hline RDW, \% & $21.30(18.10-24.40)$ & $20.25(17.70-23.70)$ & 0.217 \\
\hline Reticulocyte count,/mL & $133140(90480-173360)$ & $142055(94085-181290)$ & $0.478^{*}$ \\
\hline $\mathrm{WBC}, / \mathrm{mL}$ & $10800(8700-13000)$ & $10100(7650-13550)$ & $0.135^{*}$ \\
\hline Neutrophils, /mL & $5282(3800-6600)$ & 4348 (3350-6713) & 0.091 \\
\hline Eosinophils, /mL & $300.00(176.50-557.80)$ & $326.00(143.50-572.00)$ & 0.862 \\
\hline Lymphocytes, /mL & $3629(2842-4600)$ & $3700(2626-4514)$ & 0.591 \\
\hline Monocytes, /mL & $900.00(600.00-1300.00)$ & $805.00(563.50-1265.00)$ & 0.322 \\
\hline Platelet count, $\times 10^{3} / \mathrm{mL}$ & $379.00(281.00-474.00)$ & $383.00(271.80-462.30)$ & $0.655^{*}$ \\
\hline Platelet volume average, fL & $8.00(7.50-8.60)$ & $7.95(7.30-8.60)$ & $0.875^{*}$ \\
\hline Plateletcrit, \% & $0.29(0.23-0.36)$ & $0.28(0.21-0.36)$ & 0.375 \\
\hline \multicolumn{4}{|l|}{ Biochemical markers } \\
\hline $\mathrm{TC}, \mathrm{mg} / \mathrm{dL}$ & $127.00(111.50-145.00)$ & $116.50(100.30-138.00)$ & 0.019 \\
\hline HDL-C, mg/dL & $36.00(32.00-42.75)$ & $36.00(31.00-43.75)$ & $0.798^{*}$ \\
\hline LDL-C, mg/dL & $68.40(52.50-82.60)$ & $57.70(48.10-77.45)$ & 0.034 \\
\hline VLDL-C, mg/dL & $20.60(16.20-25.40)$ & $17.80(13.80-23.80)$ & 0.050 \\
\hline Triglycerides, mg/dL & $103.50(81.00-127.50)$ & $89.50(69.25-118.80)$ & 0.040 \\
\hline Non-HDL-C, mg/dL & $90.50(74.00-106.80)$ & $79.00(66.25-99.75)$ & 0.024 \\
\hline TC/HDL-C ratio & $3.34(2.83-4.26)$ & $3.20(2.67-3.88)$ & 0.133 \\
\hline Triglycerides/HDL-C ratio & $2.71(1.93-3.73)$ & $2.47(1.70-3.30)$ & 0.092 \\
\hline LDL-C/HDL-C ratio & $1.80(1.38-2.43)$ & $1.64(1.20-2.11)$ & 0.135 \\
\hline Total bilirubin, mg/dL & $2.07(1.26-3.18)$ & $1.86(1.22-3.14)$ & 0.573 \\
\hline Direct bilirubin, mg/dL & $0.25(0.23-0.45)$ & $0.35(0.28-0.50)$ & 0.378 \\
\hline Indirect bilirubin, $\mathrm{mg} / \mathrm{dL}$ & $1.67(0.98-2.90)$ & $1.34(0.87-2.75)$ & 0.339 \\
\hline $\mathrm{LDH}, \mathrm{U} / \mathrm{L}$ & $917.00(644.50-1304.00)$ & $852.00(614.00-1137.00)$ & 0.276 \\
\hline ALT, U/L & $14.50(11.00-20.00)$ & $14.00(10.00-19.00)$ & 0.326 \\
\hline AST, U/L & $37.50(26.00-54.75)$ & $37.00(24.25-50.75)$ & 0.535 \\
\hline Total protein, $\mathrm{g} / \mathrm{dL}$ & $8.33(8.01-8.88)$ & $8.09(7.53-8.82)$ & $0.022^{*}$ \\
\hline Albumin, g/dL & $4.79(4.60-5.05)$ & $4.74(4.53-4.94)$ & 0.158 \\
\hline Globulin, g/dL & $3.52(3.19-4.00)$ & $3.27(2.98-3.94)$ & 0.046 \\
\hline Albumin/globulin ratio & $1.35(1.20-1.50)$ & $1.41(1.22-1.62)$ & $0.147^{*}$ \\
\hline Iron, $\mathrm{mcg} / \mathrm{dL}$ & $92.00(74.00-117.00)$ & $92.00(70.25-127.00)$ & 0.795 \\
\hline Ferritin, $\eta \mathrm{g} / \mathrm{mL}$ & $144.60(98.60-200.60)$ & $177.30(72.70-300.40)$ & 0.440 \\
\hline Urea nitrogen, $\mathrm{mg} / \mathrm{dL}$ & $17.19(14.81-21.00)$ & $15.74(13.50-21.14)$ & 0.169 \\
\hline Creatinine, $\mathrm{mg} / \mathrm{dL}$ & $0.48(0.38-0.61)$ & $0.48(0.38-0.60)$ & 0.999 \\
\hline $\mathrm{CRP}, \mathrm{mg} / \mathrm{L}$ & $2.41(1.67-3.78)$ & $2.57(1.80-3.53)$ & 0.730 \\
\hline $\mathrm{AAT}, \mathrm{mg} / \mathrm{dL}$ & $71.30(37.35-118.50)$ & $69.05(38.23-118.30)$ & 0.984 \\
\hline
\end{tabular}

RBC: red blood cells; MCV: mean cell volume; MCH: mean cell hemoglobin; MCHC: mean corpuscular hemoglobin concentration; RDW: red cell distribution width; LDH: lactate dehydrogenase; WBC: white blood cells; TC: total cholesterol; HDL-C: high-density lipoprotein cholesterol; LDL-C: low-density lipoprotein cholesterol; VLDL-C: very-low-density lipoprotein cholesterol; AST: aspartate aminotransferase; ALT: alanine aminotransferase; CRP: C reactive protein; AAT: alpha 1-antitrypsin; IQR: interquartile range. Bold values indicate significance at $P<0.05$. All $P$ values obtained by the Mann-Whitney $U$ test, except for those with asterisk $(*)$, for which the independent $t$-test was used. 


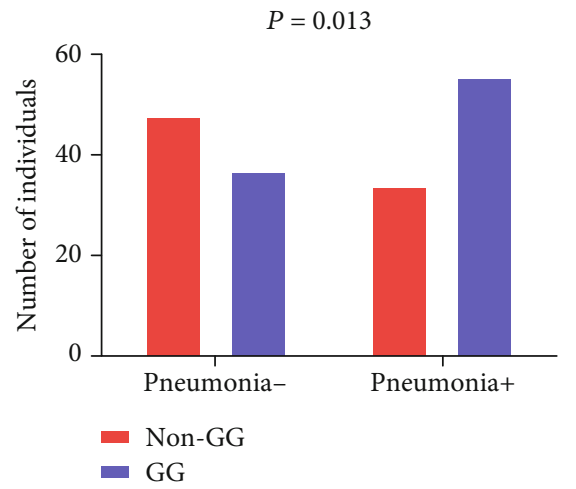

(a)

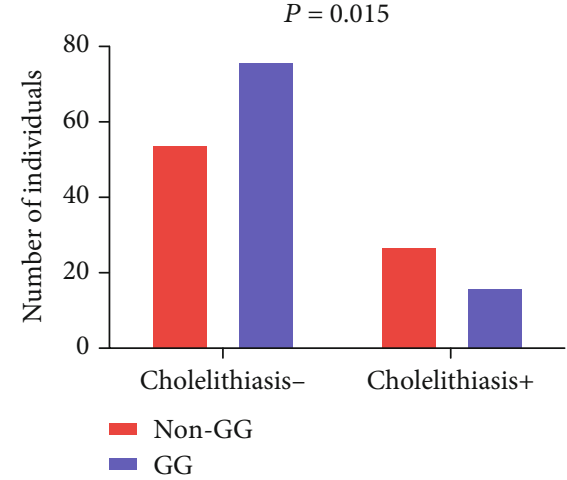

(b)

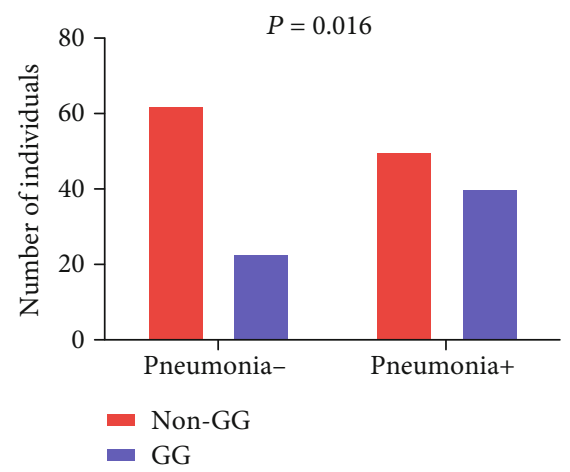

(c)

FIgURE 4: Associations between the GG and CGG haplotypes and clinical manifestations in individuals with SCD. GG haplotype was associated with (a) a previous history of pneumonia, while non-GG haplotypes were associated with (b) a previous history of cholelithiasis. The CCG haplotype was associated with (c) a previous history of pneumonia (all $P$ values obtained by the Chi-square test).

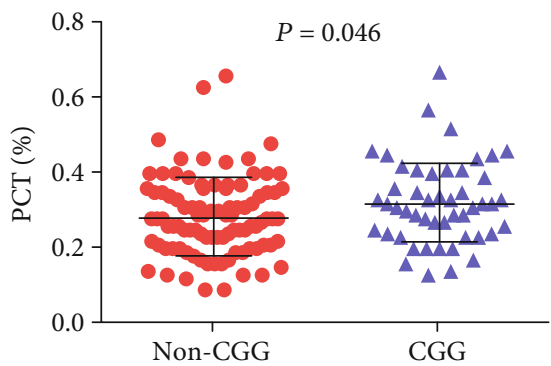

(a)

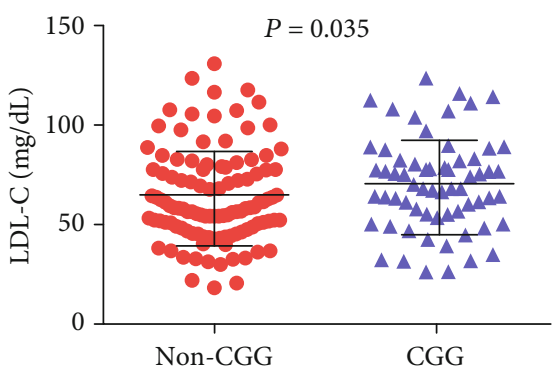

(c)

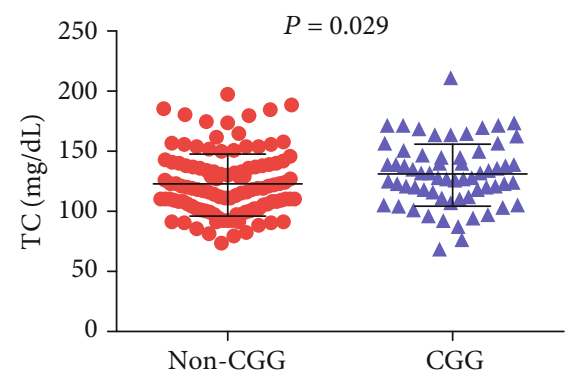

(b)

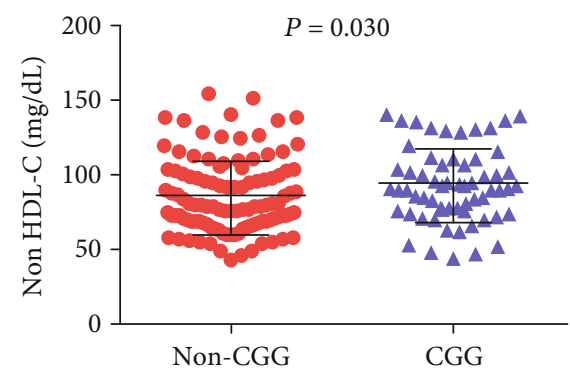

(d)

FIGURE 5: Associations between the CGG haplotype and laboratory parameters in individuals with SCD. Carriers of the CGG haplotype presented increased levels of (a) plateletcrit (PCT), (b) total cholesterol (TC), (c) low-density lipoprotein cholesterol (LDL-C), and (d) nonhigh-density lipoprotein cholesterol (non-HDL-C) ( $P$ values obtained by the Mann-Whitney $U$ test). 
TABLE 7: Association of TGFBR3 CGG haplotype with laboratory biomarkers.

\begin{tabular}{|c|c|c|c|}
\hline \multirow[b]{2}{*}{ Parameter } & \multicolumn{2}{|c|}{ TGFBR3 CGG haplotype } & \multirow[b]{2}{*}{$P$ value } \\
\hline & $\begin{array}{c}\text { CGG } \\
N=63 \\
\text { Median (IQR) }\end{array}$ & $\begin{array}{c}\text { Non-CGG } \\
N=112 \\
\text { Median (IQR) }\end{array}$ & \\
\hline \multicolumn{4}{|l|}{ Hemoglobin pattern } \\
\hline Fetal hemoglobin, \% & $4.50(1.70-8.15)$ & $5.85(1.52-11.70)$ & 0.331 \\
\hline S hemoglobin, \% & $80.70(52.00-90.53)$ & $79.70(55.35-88.20)$ & 0.676 \\
\hline \multicolumn{4}{|l|}{ Hematological markers } \\
\hline RBC, $10^{6} / \mathrm{mL}$ & $2.98(2.54-3.99)$ & $2.95(2.53-3.78)$ & 0.681 \\
\hline Hemoglobin, g/dL & $9.00(7.85-11.35)$ & $9.15(8.10-10.80)$ & 0.958 \\
\hline Hematocrit, \% & $27.00(23.70-33.50)$ & $27.40(23.58-32.10)$ & 0.977 \\
\hline $\mathrm{MCV}, \mathrm{fL}$ & $86.20(80.05-93.78)$ & $89.75(80.73-97.03)$ & 0.230 \\
\hline $\mathrm{MCH}, \rho \mathrm{g}$ & $29.35(27.13-32.38)$ & $30.25(27.40-32.68)$ & $0.242^{*}$ \\
\hline $\mathrm{MCHC}, \mathrm{g} / \mathrm{dL}$ & $33.90(33.20-34.45)$ & $33.70(33.20-34.28)$ & $0.304^{*}$ \\
\hline RDW, \% & $21.60(18.10-24.50)$ & $20.40(17.70-23.70)$ & 0.177 \\
\hline Reticulocyte count, /mL & $131100(106860-170440)$ & $139385(91350-180125)$ & 0.948 \\
\hline $\mathrm{WBC}, / \mathrm{mL}$ & $10800(9150-13000)$ & $10300(7800-13400)$ & $0.152^{*}$ \\
\hline Neutrophils, /mL & 4876 (3750-6650) & $4810(3392-6600)$ & 0.452 \\
\hline Eosinophils, /mL & $300.00(182.00-520.00)$ & $335.00(146.50-552.50)$ & 0.969 \\
\hline Lymphocytes, /mL & $3840(2943-4640)$ & $3500(2574-4440)$ & 0.090 \\
\hline Monocytes, /mL & $944.00(597.00-1346.00)$ & $840.50(575.30-1253.00)$ & 0.209 \\
\hline Platelet count, $\mathrm{x} 10^{3} / \mathrm{mL}$ & $389.00(310.50-484.50)$ & $371.50(267.30-454.80)$ & $0.171^{*}$ \\
\hline Platelet volume average, $\mathrm{fL}$ & $7.90(7.50-8.50)$ & $8.00(7.30-8.60)$ & $0.989^{*}$ \\
\hline Plateletcrit, \% & $0.32(0.24-0.40)$ & $0.28(0.21-0.35)$ & 0.046 \\
\hline \multicolumn{4}{|l|}{ Biochemical markers } \\
\hline $\mathrm{TC}, \mathrm{mg} / \mathrm{dL}$ & $128.00(112.00-147.00)$ & $119.00(103.50-138.30)$ & 0.029 \\
\hline HDL-C, mg/dL & $36.00(31.75-42.00)$ & $36.00(32.00-43.00)$ & $0.645^{*}$ \\
\hline LDL-C, mg/dL & $68.40(55.40-82.80)$ & $58.50(48.40-78.00)$ & 0.035 \\
\hline VLDL-C, mg/dL & $20.80(15.45-26.40)$ & $19.10(14.45-23.50)$ & 0.176 \\
\hline Triglycerides, mg/dL & $105.00(77.50-132.50)$ & $94.00(72.50-117.00)$ & 0.141 \\
\hline Non-HDL-C, mg/dL & $91.00(76.00-108.00)$ & $79.00(68.00-101.50)$ & 0.030 \\
\hline TC/HDL-C ratio & $3.36(2.86-4.32)$ & $3.22(2.67-3.96)$ & 0.134 \\
\hline Triglycerides/HDL-C ratio & $2.89(1.87-3.96)$ & $2.51(1.82-3.45)$ & 0.086 \\
\hline LDL-C/HDL-C ratio & $1.83(1.41-2.43)$ & $1.68(1.20-2.20)$ & 0.135 \\
\hline Total bilirubin, mg/dL & $2.08(1.26-3.14)$ & $1.92(1.25-3.20)$ & 0.657 \\
\hline Direct bilirubin, mg/dL & $0.34(0.23-0.45)$ & $0.36(0.26-0.49)$ & 0.373 \\
\hline Indirect bilirubin, $\mathrm{mg} / \mathrm{dL}$ & $1.60(0.96-2.66)$ & $1.47(0.88-2.79)$ & 0.664 \\
\hline $\mathrm{LDH}, \mathrm{U} / \mathrm{L}$ & $886.00(651.00-1261.00)$ & $855.00(614.50-1224.00)$ & 0.558 \\
\hline ALT, U/L & $15.00(11.00-18.00)$ & $14.00(10.00-20.00)$ & 0.675 \\
\hline AST, U/L & $36.50(26.00-49.50)$ & $38.00(24.00-54.00)$ & 0.855 \\
\hline Total protein, g/dL & $8.33(8.02-8.89)$ & $8.09(7.66-8.82)$ & $0.055^{*}$ \\
\hline Albumin, g/dL & $4.77(4.60-5.05)$ & $4.79(4.59-4.98)$ & $0.625^{*}$ \\
\hline Globulin, g/dL & $3.60(3.28-4.03)$ & $3.32(3.00-3.93)$ & 0.022 \\
\hline Albumin/globulin ratio & $1.31(1.20-1.50)$ & $1.40(1.26-1.61)$ & $0.059^{*}$ \\
\hline Iron, $\mathrm{mcg} / \mathrm{dL}$ & $88.00(74.25-105.00)$ & $92.00(67.50-127.50)$ & 0.363 \\
\hline Ferritin, $\eta \mathrm{g} / \mathrm{mL}$ & $142.00(98.30-219.20)$ & $155.70(73.25-254.30)$ & 0.931 \\
\hline Urea nitrogen, $\mathrm{mg} / \mathrm{dL}$ & $17.00(14.70-20.49)$ & $17.10(14.00-21.89)$ & 0.915 \\
\hline Creatinine, mg/dL & $0.48(0.38-0.59)$ & $0.49(0.38-0.61)$ & 0.605 \\
\hline $\mathrm{CRP}, \mathrm{mg} / \mathrm{L}$ & $2.43(1.59-3.78)$ & $2.37(1.80-3.53)$ & 0.780 \\
\hline $\mathrm{AAT}, \mathrm{mg} / \mathrm{dL}$ & $65.80(34.75-121.00)$ & $72.55(39.20-116.00)$ & 0.566 \\
\hline
\end{tabular}

RBC: red blood cells; MCV: mean cell volume; MCH: mean cell hemoglobin; MCHC: mean corpuscular hemoglobin concentration; RDW: red cell distribution width; LDH: lactate dehydrogenase; WBC: white blood cells; TC: total cholesterol; HDL-C: high-density lipoprotein cholesterol; LDL-C: low-density lipoprotein cholesterol; VLDL-C: very-low-density lipoprotein cholesterol; AST: aspartate aminotransferase; ALT: alanine aminotransferase; CRP: C reactive protein; AAT: alpha 1-antitrypsin; IQR: interquartile range. Bold values indicate significance at $P<0.05$. All $P$ values obtained by the Mann-Whitney $U$ test, except for those with asterisk $\left({ }^{*}\right)$, for which the independent $t$-test was used. 
TABLE 8: Association of occurrence of pneumonia with laboratory biomarkers.

\begin{tabular}{|c|c|c|c|}
\hline Parameter & $\begin{array}{c}\text { Pneumonia- } \\
N=85 \\
\text { Median (IQR) }\end{array}$ & $\begin{array}{c}\text { Pneumonia+ } \\
N=90 \\
\text { Median (IQR) }\end{array}$ & $P$ value \\
\hline \multicolumn{4}{|l|}{ Hemoglobin pattern } \\
\hline Fetal hemoglobin, \% & $4.90(1.30-8.30)$ & $6.00(1.77-11.90)$ & 0.208 \\
\hline S hemoglobin, \% & $76.20(51.70-89.50)$ & $80.95(56.75-89.20)$ & 0.347 \\
\hline \multicolumn{4}{|l|}{ Hematological markers } \\
\hline RBC, $10^{6} / \mathrm{mL}$ & $2.86(2.53-4.10)$ & $2.98(2.53-3.73)$ & 0.499 \\
\hline Hemoglobin, g/dL & $9.00(8.05-11.35)$ & $9.10(8.00-10.65)$ & 0.685 \\
\hline Hematocrit, \% & $27.00(23.45-33.35)$ & $27.45(23.93-31.95)$ & 0.760 \\
\hline $\mathrm{MCV}, \mathrm{fL}$ & $85.60(80.30-93.50)$ & $89.80(81.40-97.80)$ & 0.194 \\
\hline $\mathrm{MCH}, \rho \mathrm{g}$ & $29.30(27.20-32.45)$ & $30.50(27.40-33.10)$ & $0.365^{*}$ \\
\hline $\mathrm{MCHC}, \mathrm{g} / \mathrm{dL}$ & $33.90(33.45-34.40)$ & $33.60(33.10-34.30)$ & $0.226^{*}$ \\
\hline RDW, \% & $20.40(17.40-23.75)$ & $21.20(18.00-24.38)$ & 0.103 \\
\hline Reticulocyte count, /mL & $127880(86020-171955)$ & $145305(105170-183728)$ & $0.100^{*}$ \\
\hline $\mathrm{WBC}, / \mathrm{mL}$ & $10500(7350-13050)$ & $10600(8300-13500)$ & $0124^{*}$ \\
\hline Neutrophils, /mL & $4621(3290-6468)$ & $5060(3600-7400)$ & 0.172 \\
\hline Eosinophils, /mL & $349.00(130.00-595.50)$ & $300.00(179.00-553.00)$ & 0.781 \\
\hline Lymphocytes, /mL & $3549(2400-4490)$ & $3790(2954-4537)$ & 0.152 \\
\hline Monocytes, /mL & $804.00(600.00-1259.00)$ & $915.50(573.80-1300.00)$ & 0.412 \\
\hline Platelet count, $\mathrm{x} 10^{3} / \mathrm{mL}$ & $357.00(247.00-432.50)$ & $395.00(315.50-491.00)$ & 0.051 \\
\hline Platelet volume average, fL & $8.10(7.35-8.70)$ & $7.90(7.50-8.40)$ & $0.245 *$ \\
\hline Plateletcrit, \% & $0.28(0.20-0.35)$ & $0.30(0.24-0.40)$ & 0.062 \\
\hline \multicolumn{4}{|l|}{ Biochemical markers } \\
\hline $\mathrm{TC}, \mathrm{mg} / \mathrm{dL}$ & $117.00(102.00-138.00)$ & $128.50(110.50-150.80)$ & $0.004^{*}$ \\
\hline HDL-C, mg/dL & $37.00(31.50-42.50)$ & $35.00(32.00-43.00)$ & 0.979 \\
\hline LDL-C, mg/dL & $58.80(49.80-78.00)$ & $68.40(50.90-87.35)$ & $0.025^{*}$ \\
\hline VLDL-C, mg/dL & $18.50(14.15-23.30)$ & $20.60(15.75-25.95)$ & 0.084 \\
\hline Triglycerides, mg/dL & $92.50(70.75-116.50)$ & $103.00(79.50-131.30)$ & 0.073 \\
\hline Non-HDL-C, mg/dL & $78.00(67.50-100.00)$ & $91.00(73.00-111.00)$ & 0.012 \\
\hline TC/HDL-C ratio & $3.13(2.62-3.94)$ & $3.28(2.87-4.37)$ & 0.052 \\
\hline Triglycerides/HDL-C ratio & $2.48(1.81-3.35)$ & $2.71(1.88-4.00)$ & 0.073 \\
\hline LDL-C/HDL-C ratio & $1.67(1.16-2.20)$ & $1.77(1.40-2.43)$ & 0.184 \\
\hline Total bilirubin, mg/dL & $2.02(1.16-3.06)$ & $2.02(1.29-3.24)$ & 0.716 \\
\hline Direct bilirubin, mg/dL & $0.34(0.25-0.46)$ & $0.37(0.26-0.49)$ & 0.318 \\
\hline Indirect bilirubin, $\mathrm{mg} / \mathrm{dL}$ & $1.53(0.88-2.74)$ & $1.60(0.99-2.91)$ & 0.565 \\
\hline $\mathrm{LDH}, \mathrm{U} / \mathrm{L}$ & $866.50(605.00-1214.00)$ & $858.50(665.00-1324.00)$ & 0.326 \\
\hline ALT, U/L & $12.50(10.00-18.75)$ & $15.00(11.00-20.00)$ & 0.161 \\
\hline AST, U/L & $38.00(25.25-50.75)$ & $36.00(26.00-53.50)$ & 0.968 \\
\hline Total protein, $\mathrm{g} / \mathrm{dL}$ & $8.30(7.70-8.85)$ & $8.16(7.85-8.85)$ & $0.957^{*}$ \\
\hline Albumin, g/dL & $4.85(4.60-5.02)$ & $4.77(4.57-4.98)$ & $0.123^{*}$ \\
\hline Globulin, g/dL & $3.42(3.00-4.01)$ & $3.47(3.13-3.94)$ & 0.560 \\
\hline Albumin/globulin ratio & $1.40(1.22-1.60)$ & $1.35(1.21-1.50)$ & $0.267^{*}$ \\
\hline Iron, $\mathrm{mcg} / \mathrm{dL}$ & $92.00(70.75-127.50)$ & $92.00(72.50-108.50)$ & 0.231 \\
\hline Ferritin, $\eta \mathrm{g} / \mathrm{mL}$ & $151.50(89.75-238.20)$ & $139.30(71.70-213.40)$ & 0.466 \\
\hline Urea nitrogen, $\mathrm{mg} / \mathrm{dL}$ & $16.64(14.00-21.22)$ & $17.50(14.39-21.00)$ & 0.776 \\
\hline Creatinine, mg/dL & $0.50(0.42-0.66)$ & $0.46(0.36-0.57)$ & 0.014 \\
\hline $\mathrm{CRP}, \mathrm{mg} / \mathrm{L}$ & $2.12(1.63-2.98)$ & $3.21(1.96-4.79)$ & $<0.001$ \\
\hline $\mathrm{AAT}, \mathrm{mg} / \mathrm{dL}$ & $70.25(39.23-114.80)$ & $72.00(37.10-120.00)$ & 0.869 \\
\hline
\end{tabular}

RBC: red blood cells; MCV: mean cell volume; MCH: mean cell hemoglobin; MCHC: mean corpuscular hemoglobin concentration; RDW: red cell distribution width; LDH: lactate dehydrogenase; WBC: white blood cells; TC: total cholesterol; HDL-C: high-density lipoprotein cholesterol; LDL-C: low-density lipoprotein cholesterol; VLDL-C: very-low-density lipoprotein cholesterol; AST: aspartate aminotransferase; ALT: alanine aminotransferase; CRP: C reactive protein; AAT: alpha 1-antitrypsin; IQR: interquartile range. Bold values indicate significance at $P<0.05$. All $P$ values obtained by the Mann-Whitney $U$ test, except for those with asterisk $\left({ }^{*}\right)$, for which the independent $t$-test was used. 
TABLE 9: Association of occurrence of cholelithiasis with laboratory biomarkers.

\begin{tabular}{|c|c|c|c|}
\hline Parameter & $\begin{array}{c}\text { Cholelithiasis- } \\
N=132 \\
\text { Median (IQR) }\end{array}$ & $\begin{array}{c}\text { Cholelithiasis+ } \\
N=43 \\
\text { Median (IQR) }\end{array}$ & $P$ value \\
\hline \multicolumn{4}{|l|}{ Hemoglobin pattern } \\
\hline Fetal hemoglobin, \% & $3.50(1.20-7.50)$ & $7.50(3.60-13.75)$ & $<0.001$ \\
\hline S hemoglobin, \% & $78.70(51.93-89.35)$ & $85.40(78.35-90.45)$ & 0.002 \\
\hline \multicolumn{4}{|l|}{ Hematological markers } \\
\hline $\mathrm{RBC}, 10^{6} / \mathrm{mL}$ & $3.11(2.59-3.97)$ & $2.63(2.33-3.34)$ & 0.003 \\
\hline Hemoglobin, g/dL & $9.25(8.10-11.03)$ & $8.60(7.60-10.50)$ & 0.130 \\
\hline Hematocrit, \% & $27.50(24.00-32.55)$ & $26.00(22.70-31.80)$ & 0.176 \\
\hline $\mathrm{MCV}, \mathrm{fL}$ & $85.60(79.80-93.30)$ & $94.30(88.40-104.80)$ & $<0.001^{*}$ \\
\hline $\mathrm{MCH}, \rho \mathrm{g}$ & $28.80(26.80-31.80)$ & $32.30(30.10-35.53)$ & $<0.001^{*}$ \\
\hline $\mathrm{MCHC}, \mathrm{g} / \mathrm{dL}$ & $33.80(33.23-34.40)$ & $33.70(33.10-34.10)$ & $0.458^{*}$ \\
\hline RDW, \% & $20.80(17.73-24.15)$ & $20.80(18.10-24.00)$ & 0.999 \\
\hline Reticulocyte count, $/ \mathrm{mL}$ & $142285(99920-142285)$ & $123200(79580-157800)$ & 0.085 \\
\hline $\mathrm{WBC}, / \mathrm{mL}$ & $10800(8100-13300)$ & $9900(7400-13200)$ & $0.277^{*}$ \\
\hline Neutrophils, /mL & $5026(3600-6600)$ & $4200(3080-6600)$ & 0.096 \\
\hline Eosinophils, /mL & $345.00(163.00-597.00)$ & $288.00(100.00-444.00)$ & 0.097 \\
\hline Lymphocytes, /mL & $3658(2800-4532)$ & $3800(2652-4700)$ & 0.626 \\
\hline Monocytes, $/ \mathrm{mL}$ & $915.50(600.00-1300.00)$ & $784.00(500.00-1260.00)$ & 0.224 \\
\hline Platelet count, $\mathrm{x} 10^{3} / \mathrm{mL}$ & $390.00(289.30-476.00)$ & $352.00(238.00-462.00)$ & 0.149 \\
\hline Platelet volume average, fL & $8.10(7.50-8.60)$ & $7.90(7.20-8.60)$ & $0.173^{*}$ \\
\hline Plateletcrit, \% & $0.30(0.23-0.36)$ & $0.28(0.20-0.32)$ & 0.104 \\
\hline \multicolumn{4}{|l|}{ Biochemical markers } \\
\hline $\mathrm{TC}, \mathrm{mg} / \mathrm{dL}$ & $126.00(110.00-145.00)$ & $110.00(97.00-132.00)$ & $0.012^{*}$ \\
\hline HDL-C, mg/dL & $36.00(32.00-43.00)$ & $36.00(32.00-44.00)$ & $0.796^{*}$ \\
\hline LDL-C, mg/dL & $64.70(52.15-83.15)$ & $53.80(45.60-75.60)$ & 0.003 \\
\hline VLDL-C, mg/dL & $20.20(15.35-23.90)$ & $17.50(14.45-25.20)$ & 0.399 \\
\hline Triglycerides, mg/dL & $101.00(77.00-121.00)$ & $88.00(72.50-125.00)$ & 0.355 \\
\hline Non-HDL-C, mg/dL & $90.00(72.00-106.00)$ & $74.00(65.00-91.00)$ & 0.007 \\
\hline TC/HDL-C ratio & $3.38(2.78-4.24)$ & $2.97(2.67-3.23)$ & $<0.001$ \\
\hline Triglycerides/HDL-C ratio & $2.68(1.87-3.60)$ & $2.17(1.76-3.16)$ & 0.110 \\
\hline LDL-C/HDL-C ratio & $1.83(1.38-2.43)$ & $1.41(1.16-1.76)$ & $<0.001$ \\
\hline Total bilirubin, mg/dL & $1.89(1.18-3.00)$ & $2.79(1.54-3.91)$ & 0.016 \\
\hline Direct bilirubin, mg/dL & $0.35(0.27-0.49)$ & $0.36(0.24-0.44)$ & 0.880 \\
\hline Indirect bilirubin, $\mathrm{mg} / \mathrm{dL}$ & $1.44(0.88-2.61)$ & $2.17(1.21-3.50)$ & 0.010 \\
\hline $\mathrm{LDH}, \mathrm{U} / \mathrm{L}$ & $879.00(618.00-1269.00)$ & $855.00(673.00-1153.00)$ & 0.873 \\
\hline ALT, U/L & $14.00(10.25-19.00)$ & $15.00(10.00-19.00)$ & 0.779 \\
\hline AST, U/L & $39.00(26.00-54.00)$ & $35.00(27.00-52.00)$ & 0.512 \\
\hline Total protein, g/dL & $8.21(7.85-8.85)$ & $8.44(7.76-9.00)$ & $0.215^{*}$ \\
\hline Albumin, g/dL & $4.79(4.60-4.97)$ & $4.79(4.57-5.00)$ & 0.868 \\
\hline Globulin, g/dL & $3.43(3.10-3.90)$ & $3.50(3.03-4.11)$ & $0.200^{*}$ \\
\hline Albumin/globulin ratio & $1.39(1.25-1.55)$ & $1.30(1.12-1.62)$ & $0.380^{*}$ \\
\hline Iron, $\mathrm{mcg} / \mathrm{dL}$ & $92.00(74.00-120.80)$ & $94.00(67.00-124.00)$ & 0.960 \\
\hline Ferritin, $\eta \mathrm{g} / \mathrm{mL}$ & $154.40(72.70-248.00)$ & $148.90(89.33-410.80)$ & 0.577 \\
\hline Urea nitrogen, $\mathrm{mg} / \mathrm{dL}$ & $18.00(14.53-21.62)$ & $15.00(12.76-17.22)$ & $0.002^{*}$ \\
\hline Creatinine, $\mathrm{mg} / \mathrm{dL}$ & $0.48(0.38-0.63)$ & $0.48(0.36-0.57)$ & 0.380 \\
\hline $\mathrm{CRP}, \mathrm{mg} / \mathrm{L}$ & $2.37(1.71-3.71)$ & $2.75(1.87-4.71)$ & 0.128 \\
\hline $\mathrm{AAT}, \mathrm{mg} / \mathrm{dL}$ & $68.20(37.10-120.00)$ & $73.20(39.65-100.20)$ & 0.873 \\
\hline
\end{tabular}

RBC: red blood cells; MCV: mean cell volume; MCH: mean cell hemoglobin; MCHC: mean corpuscular hemoglobin concentration; RDW: red cell distribution width; LDH: lactate dehydrogenase; WBC: white blood cells; TC: total cholesterol; HDL-C: high-density lipoprotein cholesterol; LDL-C: low-density lipoprotein cholesterol; VLDL-C: very-low-density lipoprotein cholesterol; AST: aspartate aminotransferase; ALT: alanine aminotransferase; CRP: C reactive protein; AAT: alpha 1-antitrypsin; IQR: interquartile range. Bold values indicate significance at $P<0.05$. All $P$ values obtained by the Mann-Whitney $U$ test, except for those with asterisk $\left({ }^{*}\right)$, for which the independent $t$-test was used. 
in SCA vasculopathy, marked by enhanced oxidative stress, low NO bioavailability in addition to endothelial dysfunction; however, it is important to emphasize that in SCD there is no formation of atheroma plaques $[4,46]$. Vasculopathy is responsible for several clinical manifestations in SCD, such as stroke, priapism, pulmonary hypertension, and leg ulcers [4].

Moreover, high triglyceride and non-HDL-C levels have also been associated to atherosclerosis. In the lipolysis of triglycerides, triglyceride-rich remnants are released in vessels contributing to increase inflammation, coagulation, and endothelial dysfunction [47]. Increased triglyceride levels were shown to be a potential risk factor for pulmonary hypertension, a frequent respiratory complication in individuals with SCD [29].

Non-HDL-C is a cholesterol carried by apolipoprotein B, including those carried by LDL-C and VLDL-C, and is considered a useful marker of atherosclerosis [48]. Previous studies performed in individuals with cardiovascular disease reported high non-HDL-C levels $[49,50]$; however, no significant alterations in non-HDL-C levels were found in SCD individuals [32].

TC, LDL-C, triglycerides, and non-HDL-C are markers of the lipid metabolism associated with inflammation related to vascular response $[45,46,48]$. The TGF- $\beta$ pathway has been associated with vascular inflammatory responses in several diseases. In many cases, cholesterol uptake and trafficking are also responsible for vessel wall modifications, which can contribute to inflammation [51, 52]. A previous study reported that TGF- $\beta$ signaling regulates lipid metabolism through the induction of lipogenesis genes resulting in increased triglyceride synthesis and lipid accumulation. TGF- $\beta$ signaling activates SMAD proteins, triggering the activation of these pathways. The inhibition of these pathways suppresses changes in gene expression associated with lipid metabolism [53]. Therefore, the identification of markers, which may modulate the endothelial inflammatory response in SCD is relevant to monitor the clinical course and may contribute to understand the disease pathophysiology.

TGFBRIII, encoded by the TGFBR3 gene, plays an important role in regulating and mediating the signal transduction of TGF- $\beta$ [54]. In the literature, polymorphisms in TGFBR3 have been associated with inflammatory diseases other than SCD, such as Marfan syndrome, bladder cancer, and Behçet's disease [14, 16-18]. Marfan syndrome presents as clinical complications in the cardiovascular, skeletal, pulmonary, ocular, and nervous system; its physiopathology includes alterations in extracellular matrix deposition in vessels, similar to findings reported in SCD [16]. Behçet's disease is characterized by multisystemic vasculitis with marked inflammatory lesions in the central nervous system, skin, joints, orogenital mucosa, and eyes; analogous to SCD, the inflammatory component is the main physiopathological feature of this disease [18].

Individuals with the GG haplotype presented high levels of total proteins and globulin, which is similar to previous reports in individuals with SCD compared to controls [55, 56]. This hyperproteinemia arises from hyperglobulinemia, which is known to occur in SCD individuals as a result of erythrocyte destruction during sickling [57]. Thus, it is expected that individuals with the GG haplotype would present more prominent hemolysis than those with non-GG haplotypes. High levels of total protein were also identified in individuals with GG genotype of rs2038931 polymorphism when compared to A_ genotypes.

With regard to clinical manifestations, the haplotypes have showed to be more informative than the polymorphisms individually analyzed; both the GG and CGG haplotypes of gene TGFBR3 were associated with the occurrence of pneumonia. In a large cohort of SCD individuals, pneumonia was one of the leading causes of hospitalization, second only to sickle cell crisis [58]. A previous study performed in individuals with SCA, the majority of whom presented Streptococcus pneumoniae infection, identified that a polymorphism in TGFBR3 was associated with increased susceptibility to bacteremia [34]. Streptococcus pneumoniae infection is one of the main causes of pneumonia in individuals with SCD [34]. It is important to note that all individuals in this study were immunized against pneumococcal disease; therefore, it is possible that these children could have been undergoing a process of autosplenectomy, which consequently increases susceptibility to serious infections [59].

Other respiratory complications, such as asthma and bronchopulmonary dysplasia, have also been associated with TGFBR3 in the literature $[36,60]$. Similarities exist in the pathogenesis of asthma, bronchopulmonary dysplasia, and SCD, which is marked by inflammation and the activation of several cytokines, including TGF- $\beta[36,60]$.

We found that individuals with a previous history of pneumonia presented increased TC, LDL-C, non-HDL-C, CRP levels, and decreased creatinine. A previous study performed in individuals with SCD identified that the occurrence of pneumonia is related to increased frequency of other respiratory complications, such as acute chest syndrome (ACS), arising from fat embolism and pathogenic infection [61]. In a cohort without SCD, high cholesterol levels were associated with death related to a miscellaneous of respiratory diseases in a large study involving adults [62].

Serum CRP levels are clinically used to differentiate pneumonia from other acute respiratory infections [63]. In SCD, community-acquired pneumonia triggers inflammatory response and lung injury [64]. Alterations in CRP levels and other immunological markers were found in SCA individuals with lung dysfunction deriving from ACS [65]. High creatinine levels were also described as a severity marker of community-acquired pneumonia; however, in our results, the individuals with SCD and previous history of pneumonia presented lower creatinine levels [66].

We also found that non-GG haplotypes were associated with the occurrence of cholelithiasis, one of the most frequent SCD complications that occurs in $26-58 \%$ of patients. In addition, an individual with a previous history of cholelithiasis presented more prominent hemolysis than those without history. This is often associated with increased bilirubin levels, and consequently with hemolysis, and has been described as part of the hemolysis-endothelial dysfunction subphenotype $[4,67,68]$. Cholelithiasis, a clinical manifestation of SCD related to chronic hemolysis, triggers bilirubin 
production, leading to the formation of gallstones [69]. The upregulation of the TGF- $\beta$ pathway was previously detected in the gallbladders of individuals with cholelithiasis [70]. Moreover, an investigation of gene expression in patients undergoing cholecystectomy found significant expression of TGF- $\beta$ receptor (TGF $\beta$ R) I and TGF $\beta$ RII during chronic cholelithiasis in comparison to acute cholelithiasis. However, no significant increase in TGF $\beta$ RIII expression was found [71].

Altogether, our results suggest that TGFBR3 haplotypes seem to be related to inflammation and the occurrence of pneumonia. Inflammation is a physiopathological mechanism present in SCD, highlighting the relevance investigating novel biomarkers of disease severity in the clinical management of individuals with SCD. To the best of our knowledge, the present study is the first attempt to demonstrate associations between TGFBR3 haplotypes and hematological and biochemical parameters, as well as clinical manifestations in SCD.

\section{Conclusion}

Collectively, the present findings suggest that individuals with the GG and CGG haplotypes of TGFBR3 present significant lipid profile alterations and could be associated with the occurrence of pneumonia, while the non-GG haplotypes was associated with the occurrence of cholelithiasis. Further studies are essential to evaluate TGFBR3 haplotypes as prognostic markers and identify possible therapeutic targets in SCD individuals.

\section{Data Availability}

All relevant data used to support the findings of this study are included within the article and the supplementary information file.

\section{Disclosure}

The sponsors of this study, who played no role in gathering, analyzing, or interpreting the data presented herein, are public organizations whose role is to support science in general.

\section{Conflicts of Interest}

The authors declare that there is no conflict of interest regarding the publication of this paper.

\section{Acknowledgments}

We would like to thank all the SCD individuals who agreed to participate in our research protocol. We also thank the staff of the Bahia State Hematology and Hemotherapy Foundation (HEMOBA) for their assistance with sample collection and for caring for SCD individuals. We are grateful to Andris K. Walter for assistance with English language revision and manuscript copyediting services. This study was financed in part by the Coordenação de Aperfeiçoamento de Pessoal de Nível Superior-Brasil (CAPES)_-(Finance Code 001) (RPS, SCMAY and SPC). The study was also supported by the Con- selho Nacional de Desenvolvimento Científico e Tecnológico (CNPq) (470959/2014-2 and 405595/2016-6) through a grant to MSG.

\section{Supplementary Materials}

Supplementary Table 1 in the Supplementary Material for comprehensive data analysis. (Supplementary Materials)

\section{References}

[1] C. Hoppe, E. Jacob, L. Styles, F. Kuypers, S. Larkin, and E. Vichinsky, "Simvastatin reduces vaso-occlusive pain in sickle cell anaemia: a pilot efficacy trial," British Journal of Haematology, vol. 177, no. 4, pp. 620-629, 2017.

[2] C. C. da Guarda, R. P. Santiago, L. M. Fiuza et al., "Hememediated cell activation: the inflammatory puzzle of sickle cell anemia," Expert Review of Hematology, vol. 10, no. 6, pp. 533$541,2017$.

[3] T. N. Pitanga, R. R. Oliveira, D. L. Zanette et al., "Sickle red cells as danger signals on proinflammatory gene expression, leukotriene B4 and interleukin-1 beta production in peripheral blood mononuclear cell," Cytokine, vol. 83, pp. 75-84, 2016.

[4] G. J. Kato, M. T. Gladwin, and M. H. Steinberg, "Deconstructing sickle cell disease: reappraisal of the role of hemolysis in the development of clinical subphenotypes," Blood Reviews, vol. 21, no. 1, pp. 37-47, 2007.

[5] T. N. Williams and S. L. Thein, "Sickle cell anemia and its phenotypes," Annual Review of Genomics and Human Genetics, vol. 19, no. 1, pp. 113-147, 2018.

[6] G. J. Kato, V. McGowan, R. F. Machado et al., "Lactate dehydrogenase as a biomarker of hemolysis-associated nitric oxide resistance, priapism, leg ulceration, pulmonary hypertension, and death in patients with sickle cell disease," Blood, vol. 107, no. 6, pp. 2279-2285, 2006.

[7] E. P. Vichinsky, L. A. Styles, L. H. Colangelo et al., "Acute chest syndrome in sickle cell disease: clinical presentation and course," Blood, vol. 89, no. 5, pp. 1787-1792, 1997.

[8] E. P. Vichinsky, L. D. Neumayr, A. N. Earles et al., "Causes and outcomes of the acute chest syndrome in sickle cell disease. National Acute Chest Syndrome Study Group," The New England Journal of Medicine, vol. 342, no. 25, pp. 1855-1865, 2000.

[9] D. R. Powars, L. S. Chan, A. Hiti, E. Ramicone, and C. Johnson, "Outcome of sickle cell anemia: a 4-decade observational study of 1056 patients," Medicine, vol. 84, no. 6, pp. 363-376, 2005.

[10] G. J. Kato, F. B. Piel, C. D. Reid et al., "Sickle cell disease," Nature Reviews Disease Primers, vol. 4, no. 1, article 18010, 2018.

[11] S. C. Taylor, S. J. Shacks, Z. Qu, and P. Wiley, "Type 2 cytokine serum levels in healthy sickle cell disease patients," Journal of the National Medical Association, vol. 89, no. 11, pp. 753757, 1997.

[12] W. Vilas-Boas, B. A. Veloso Cerqueira, C. V. B. Figueiredo et al., "Association of homocysteine and inflammatoryrelated molecules in sickle cell anemia," Hematology, vol. 21, no. 2, pp. 126-131, 2015.

[13] M. O. S. Carvalho, T. Araujo-Santos, J. H. O. Reis et al., "Inflammatory mediators in sickle cell anaemia highlight the difference between steady state and crisis in paediatric patients," British Journal of Haematology, vol. 182, no. 6, pp. 933-936, 2018. 
[14] M. H. Steinberg and P. Sebastiani, "Genetic modifiers of sickle cell disease," American Journal of Hematology, vol. 87, no. 8, pp. 795-803, 2012.

[15] X. Gao, H. Y. Lee, E. L. da Rocha et al., "TGF- $\beta$ inhibitors stimulate red blood cell production by enhancing self-renewal of BFU-E erythroid progenitors," Blood, vol. 128, no. 23, pp. 2637-2641, 2016.

[16] M. E. Groeneveld, N. Bogunovic, R. J. P. Musters et al., "Betaglycan (TGFBR3) up-regulation correlates with increased TGF- $\beta$ signaling in Marfan patient fibroblasts in vitro," Cardiovascular Pathology: the Official Journal of the Society for Cardiovascular Pathology, vol. 32, pp. 44-49, 2018.

[17] X.-L. Liu, K. Xiao, B. Xue et al., "Dual role of TGFBR3 in bladder cancer," Oncology Reports, vol. 30, no. 3, pp. 1301-1308, 2013.

[18] R. J. Barry, J. A. Alsalem, J. Faassen, P. I. Murray, S. J. Curnow, and G. R. Wallace, "Association analysis of TGFBR3 gene with Behçet's disease and idiopathic intermediate uveitis in a Caucasian population," British Journal of Ophthalmology, vol. 99, no. 5, pp. 696-699, 2015.

[19] P. Sebastiani, M. F. Ramoni, V. Nolan, C. T. Baldwin, and M. H. Steinberg, "Genetic dissection and prognostic modeling of overt stroke in sickle cell anemia," Nature Genetics, vol. 37, no. 4, pp. 435-440, 2005.

[20] J. M. Flanagan, D. M. Frohlich, T. A. Howard et al., "Genetic predictors for stroke in children with sickle cell anemia," Blood, vol. 117, no. 24, pp. 6681-6684, 2011.

[21] V. G. Nolan, A. Adewoye, C. Baldwin et al., "Sickle cell leg ulcers: associations with haemolysis and SNPs in Klotho, TEK and genes of the TGF-beta/BMP pathway," British Journal of Haematology, vol. 133, no. 5, pp. 570-578, 2006.

[22] A. E. Ashley-Koch, L. Elliott, M. E. Kail et al., "Identification of genetic polymorphisms associated with risk for pulmonary hypertension in sickle cell disease," Blood, vol. 111, no. 12, pp. 5721-5726, 2008.

[23] L. Elliott, A. E. Ashley-Koch, L. D. Castro et al., "Genetic polymorphisms associated with priapism in sickle cell disease," British Journal of Haematology, vol. 137, no. 3, pp. 262-267, 2007.

[24] C. Baldwin, V. G. Nolan, D. F. Wyszynski et al., "Association of klotho, bone morphogenic protein 6, and annexin A2 polymorphisms with sickle cell osteonecrosis," Blood, vol. 106, no. 1, pp. 372-375, 2005.

[25] C. Martinez-Castaldi, V. G. Nolan, C. T. Baldwin, L. A. Farrer, M. H. Steinberg, and E. S. Klings, "Association of genetic polymorphisms in the TGF- $\beta$ pathway with the acute chest syndrome of sickle cell anemia," Blood, vol. 110, no. 11, p. 2247, 2007.

[26] W. T. Friedewald, R. I. Levy, and D. S. Fredrickson, "Estimation of the concentration of low-density lipoprotein cholesterol in plasma, without use of the preparative ultracentrifuge," Clinical Chemistry, vol. 18, no. 6, pp. 499502, 1972.

[27] R. Gharakhanlou, B. Farzad, H. Agha-Alinejad, L. M. Steffen, and M. Bayati, "Medidas antropométricas como preditoras de fatores de risco cardiovascular na população urbana do Irã," Arquivos Brasileiros de Cardiologia, vol. 98, no. 2, pp. 126-135, 2012.

[28] I. Lemieux, B. Lamarche, C. Couillard et al., "Total cholesterol/HDL cholesterol ratio vs LDL cholesterol/HDL cholesterol ratio as indices of ischemic heart disease risk in men," Archives of Internal Medicine, vol. 161, no. 22, pp. 2685-2692, 2001.
[29] S. Zorca, L. Freeman, M. Hildesheim et al., "Lipid levels in sickle-cell disease associated with haemolytic severity, vascular dysfunction and pulmonary hypertension," British Journal of Haematology, vol. 149, no. 3, pp. 436-445, 2010.

[30] G. Reaven, “Metabolic syndrome," Circulation, vol. 106, no. 3, pp. 286-288, 2002.

[31] R. S. Teixeira, M. B. Arriaga, R. Terse-Ramos et al., "Higher values of triglycerides: HDL-cholesterol ratio hallmark disease severity in children and adolescents with sickle cell anemia," Brazilian Journal of Medical and Biological Research, vol. 52, no. 10, article e8833, 2019.

[32] R. K. D. Ephraim, P. Adu, E. Ake et al., "Normal non-HDL cholesterol, low total cholesterol, and HDL cholesterol levels in sickle cell disease patients in the steady state: a casecontrol study of Tema Metropolis," Journal of Lipids, vol. 2016, Article ID 7650530, 5 pages, 2016.

[33] J. C. Barrett, B. Fry, J. Maller, and M. J. Daly, "Haploview: analysis and visualization of LD and haplotype maps," Bioinformatics (Oxford, England), vol. 21, no. 2, pp. 263-265, 2005.

[34] A. H. Adewoye, V. G. Nolan, Q. Ma et al., "Association of polymorphisms of IGF1R and genes in the transforming growth factor- beta/bone morphogenetic protein pathway with bacteremia in sickle cell anemia," Clinical Infectious Diseases: an Official Publication of the Infectious Diseases Society of America, vol. 43, no. 5, pp. 593-598, 2006.

[35] K. Y. Fertrin and F. F. Costa, "Genomic polymorphisms in sickle cell disease: implications for clinical diversity and treatment," Expert Review of Hematology, vol. 3, no. 4, pp. 443-458, 2014.

[36] H.-K. Kim, T.-W. Jang, M.-H. Jung et al., “Association between Genetic Variations of the Transforming Growth Factor $\beta$ Receptor Type III and Asthma in a Korean Population," Experimental \& molecular medicine, vol. 42, no. 6, pp. 420427, 2010.

[37] C. C. da Guarda, S. C. M. A. Yahouédéhou, R. P. Santiago et al., "Sickle cell disease: a distinction of two most frequent genotypes (HbSS and HbSC)," PLoS One, vol. 15, no. 1, article e0228399, 2020.

[38] M. O. Seixas, L. C. Rocha, M. B. Carvalho et al., "Levels of high-density lipoprotein cholesterol (HDL-C) among children with steady-state sickle cell disease," Lipids in Health and Disease, vol. 9, no. 1, p. 91, 2010.

[39] M. S. West, D. Wethers, J. Smith, and M. Steinberg, "Laboratory profile of sickle cell disease: a cross-sectional analysis. The cooperative study of sickle cell disease," Journal of Clinical Epidemiology, vol. 45, no. 8, pp. 893-909, 1992.

[40] M. M. Aleluia, T. C. C. Fonseca, R. Q. Souza et al., "Comparative study of sickle cell anemia and hemoglobin SC disease: clinical characterization, laboratory biomarkers and genetic profiles," BMC Hematology, vol. 17, no. 1, p. 15, 2017.

[41] T. Santiago-Sim, S. Mathew-Joseph, H. Pannu et al., "Sequencing of TGF-beta pathway genes in familial cases of intracranial aneurysm," Stroke, vol. 40, no. 5, pp. 1604-1611, 2009.

[42] C. P. Hersh, N. N. Hansel, K. C. Barnes et al., "Transforming growth factor-beta receptor-3 is associated with pulmonary emphysema," American Journal of Respiratory Cell and Molecular Biology, vol. 41, no. 3, pp. 324-331, 2009.

[43] Y. Chen, P. Yang, F. Li et al., "Association analysis of TGFBR3 gene with Vogt-Koyanagi-Harada disease and Behcet's disease in the Chinese Han population," Current Eye Research, vol. 37, no. 4, pp. 312-317, 2012. 
[44] Z. Xin, W. Zhang, A. Xu et al., "Polymorphisms in the potential functional regions of the TGF- $\beta 1$ and TGF- $\beta$ receptor genes and disease susceptibility in HBV-related hepatocellular carcinoma patients," Molecular Carcinogenesis, vol. 51, Supplement 1, pp. E123-E131, 2012.

[45] A. R. Tall and L. Yvan-Charvet, "Cholesterol, inflammation and innate immunity," Nature Reviews Immunology, vol. 15, no. 2, pp. 104-116, 2015.

[46] P. Libby, P. M. Ridker, and G. K. Hansson, "Progress and challenges in translating the biology of atherosclerosis," Nature, vol. 473, no. 7347, pp. 317-325, 2011.

[47] Y. Handelsman and M. D. Shapiro, "Triglycerides, atherosclerosis, and cardiovascular outcome studies: focus on omega-3 fatty acids," Endocrine Practice, vol. 23, no. 1, pp. 100-112, 2017.

[48] Y. Zhang, N. Q. Wu, S. Li et al., "Non-HDL-C is a better predictor for the severity of coronary atherosclerosis compared with LDL-C," Heart, Lung \& Circulation, vol. 25, no. 10, pp. 975-981, 2016.

[49] J. Liu, C. Sempos, R. P. Donahue, J. Dorn, M. Trevisan, and S. M. Grundy, "Joint distribution of non-HDL and LDL cholesterol and coronary heart disease risk prediction among individuals with and without diabetes," Diabetes Care, vol. 28, no. 8, pp. 1916-1921, 2005.

[50] S. M. Boekholdt, B. J. Arsenault, S. Mora et al., "Association of LDL cholesterol, non-HDL cholesterol, and apolipoprotein B levels with risk of cardiovascular events among patients treated with statins: a meta-analysis," JAMA, vol. 307, no. 12, pp. 1302-1309, 2012.

[51] R. C. Salter, P. Foka, T. S. Davies et al., "The role of mitogenactivated protein kinases and sterol receptor coactivator-1 in TGF- $\beta$-regulated expression of genes implicated in macrophage cholesterol uptake," Scientific Reports, vol. 6, no. 1, article $34368,2016$.

[52] J. C. Thompson, P. G. Wilson, A. P. Wyllie, A. K. Wyllie, and L. R. Tannock, "Elevated circulating TGF- $\beta$ is not the cause of increased atherosclerosis development in biglycan deficient mice," Atherosclerosis, vol. 268, pp. 68-75, 2018.

[53] L. Yang, Y. S. Roh, J. Song et al., "Transforming growth factor beta signaling in hepatocytes participates in steatohepatitis through regulation of cell death and lipid metabolism in mice," Hepatology, vol. 59, no. 2, pp. 483-495, 2014.

[54] G. C. Blobe, W. P. Schiemann, M. C. Pepin et al., "Functional roles for the cytoplasmic domain of the type III transforming growth factor $\beta$ receptor in regulating transforming growth factor $\beta$ signaling," The Journal of Biological Chemistry, vol. 276, no. 27, pp. 24627-24637, 2001.

[55] S. Pandey, A. Sharma, S. Dahia et al., "Biochemical indicator of sickle cell disease: preliminary report from India," Indian Journal of Clinical Biochemistry, vol. 27, no. 2, pp. 191-195, 2012.

[56] N. Ozgunes, Y. Oztas, S. Unal, and H. Yaman, "Structural modification of plasma albumin in sickle cell anemia," Acta Haematologica, vol. 133, no. 1, pp. 67-69, 2014.

[57] E. M. Adu, R. E. Okosun, E. N. Bini, and E. A. Ophori, "Effects of the sickle cell (S) gene on serum protein profile," Continental Journal of Biomedical Sciences, vol. 6, pp. 1-5, 2012.

[58] D. C. Brousseau, P. L. Owens, A. L. Mosso, J. A. Panepinto, and C. A. Steiner, "Acute care utilization and rehospitalizations for sickle cell disease," JAMA, vol. 303, no. 13, pp. 1288-1294, 2010.

[59] J. A. P. Braga, "Medidas gerais no tratamento das doenças falciformes," Revista Brasileira de Hematologia e Hemoterapia, vol. 29, no. 3, pp. 233-238, 2007.
[60] A. Pozarska, G. Niess, W. Seeger, and R. Morty, "A role for the accessory type III transforming growth factor $\beta$ receptor (Tgfbr 3) in lung alveolarisation," European Respiratory Journal, vol. 48, article PA4026, Supplement 60, 2016.

[61] E. P. Vichinsky, L. D. Neumayr, A. N. Earles et al., "Causes and outcomes of the acute chest syndrome in sickle cell disease," New England Journal of Medicine, vol. 342, no. 25, pp. 1855$1865,2000$.

[62] C. Iribarren, Jacobs DR Jr, S. Sidney et al., "Serum total cholesterol and risk of hospitalization, and death from respiratory disease," International Journal of Epidemiology, vol. 26, no. 6, pp. 1191-1202, 1997.

[63] A. Ruiz-González, L. Utrillo, S. Bielsa, M. Falguera, and J. M. Porcel, "The diagnostic value of serum C-reactive protein for identifying pneumonia in hospitalized patients with acute respiratory symptoms," Journal of Biomarkers, vol. 2016, Article ID 2198745, 5 pages, 2016.

[64] A. C. Miller and M. T. Gladwin, "Pulmonary complications of sickle cell disease," American Journal of Respiratory and Critical Care Medicine, vol. 185, no. 11, pp. 1154-1165, 2012.

[65] S. A. Adegoke, B. P. Kuti, K. O. Omole, O. S. Smith, O. A. Oyelami, and O. O. Adeodu, "Acute chest syndrome in sickle cell anaemia: higher serum levels of interleukin-8 and highly sensitive C-reactive proteins are associated with impaired lung function," Paediatrics and International Child Health, vol. 38, no. 4, pp. 244-250, 2018.

[66] J. Sloan, J. Wilson, C. Griffin, M. Wilkie, J. Chalmers, and S. Schembri, "Elevated creatinine is a sensitive severity marker in community acquired pneumonia," European Respiratory Journal, vol. 40, article P2506, Supplement 56, 2012.

[67] E. C. Ebert, M. Nagar, and K. D. Hagspiel, "Gastrointestinal and hepatic complications of sickle cell disease," Clinical Gastroenterology and Hepatology: The Official Clinical Practice Journal of The American Gastroenterological Association, vol. 8, no. 6, pp. 483-489, 2010.

[68] L. R. Bond, S. R. Hatty, M. E. Horn, M. Dick, H. B. Meire, and A. J. Bellingham, "Gall stones in sickle cell disease in the United Kingdom," British Medical Journal (Clinical Research Ed.), vol. 295, no. 6592, pp. 234-236, 1987.

[69] R. A. Martins, et al.R. S. Soares, F. B. D. Vito et al., "Cholelithiasis and its complications in sickle cell disease in a university hospital," Revista Brasileira de Hematologia e Hemoterapia, vol. 39, no. 1, pp. 28-31, 2017.

[70] J. Köninger, F. F. di Mola, P. di Sebastiano et al., "Transforming growth factor-beta pathway is activated in cholecystolithiasis," Langenbeck's Archives of Surgery, vol. 390, no. 1, pp. 2128, 2005.

[71] Y. D. Walawalkar, Y. Vaidya, and V. Nayak, "Alteration in transforming growth factor- $\beta$ receptor expression in gallbladder disease: implications of chronic cholelithiasis and chronic Salmonella typhi infection," Gastroenterology Insights, vol. 7, no. $1,2016$. 